\title{
REFLECTION, REMOVABLE SINGULARITIES, AND APPROXIMATION FOR PARTIAL DIFFERENTIAL EQUATIONS. II
}

\author{
LEON EHRENPREIS
}

\begin{abstract}
Let $\Omega^{j}$ be domains in $R^{n}$. For each $j$ we are given a system $\mathbf{D}^{j}$ of linear constant coefficient operators and a function $f^{j}$ on $\Omega^{j}$ satisfying $\mathbf{D}^{j} f^{j}=0$. When the $f^{j}$ satisfy certain compatibility conditions on the intersections $\Omega^{j} \cap \Omega^{j^{\prime}}$ then we can extend them so as to be solutions of $\mathbf{D}^{j}$ on larger domains. As a consequence of our methods we are able to sharpen Hartogs' theorems to allow for continuation of solutions of overdetermined systems over noncompact sets.
\end{abstract}

1. Introduction. In Part I of this series (see [1]) we introduced the following general problem: Let $\Omega^{1}, \ldots, \Omega^{l}$ be regions in $R^{n}$ containing a common set $Y$ in their closures. Consider $\mathbf{D}^{1}, \ldots, \mathbf{D}^{l}$ where $\mathbf{D}^{j}=\left(D_{1}^{j}, \ldots, D_{r_{j}}^{j}\right)$, the $D_{k}^{j}$ being linear partial differential operators with constant coefficients. [It is allowed that $\Omega^{j}=\Omega^{j^{\prime}}$ or $\mathbf{D}^{k}=\mathbf{D}^{k^{\prime}}$ for some pairs $\left(j, j^{\prime}\right)$ or $\left(k, k^{\prime}\right)$.] We assume that $Y$ can be used to set up a single parametrization (Cauchy) problem for solutions of $\mathbf{D}^{j} f^{j}=0$ for every $j$. This means that there are linear constant coefficient differential operators $h_{s}$ and subsets $Y^{s}$ of $Y$ so that any solution of $\mathbf{D}^{j} f=0$ is determined by the set of restrictions of $h_{s} f$ to $Y^{s}$ and, moreover, the map

$$
f \rightarrow\left\{\left.h_{s} f\right|_{Y^{s}}\right\}=c(f)
$$

is an isomorphism on suitable function spaces. (The $h_{s}$ are not tangential to $Y^{s}$.)

It is to be emphasized that $h_{s}$ and $Y^{s}$ do not depend on $j$. Thus, through the data $c(f)$ we can compare solutions of $\mathbf{D}^{j} f^{j}=0$ on $\Omega^{j}$ for various $j$.

Suppose we are given relations of the form

$$
\sum e_{k j}^{s} h_{s} f^{j}=0 \text { on } Y^{k}
$$

where the $e_{k j}^{s}$ are constants and for all $s$ that appear in a given sum in (1.2) we have $Y^{k} \subset Y^{s}$. What is special about the solutions $f^{j}$ ? In particular, can $f^{j}$ be extended to solutions of $\mathbf{D}^{j}$ on larger sets (removable singularities property)? Can they be approximated by solutions on larger sets (Runge property)? We shall refer to these properties as star extension and star approximation because we think of the $\Omega^{j}$ as forming a star of $Y$ in the sense of combinatorial topology.

Received by the editors June $18,1986$.

1980 Mathematics Subject Classification (1985 Revision). Primary 35B60; Secondary 32A15.

Key words and phrases. Reflection, removable singularities, Hartogs theorem, Lewy theorem.

Work partially supported by National Science Foundation grant. 
For most of our work we shall make the technically simplifying assumption that all $Y^{s}=Y$. Presumably this does not play an essential role in our work. Call $q=$ number of $h_{s}$.

Part I of this series dealt with the case when there are two sets $\Omega^{j}$ which are half-spaces whose closures intersect in $Y$. Moreover we assumed that $\mathbf{D}^{1}=\mathbf{D}^{2}$ and that equation (1.2) is of the form

$$
\text { Cauchy data } f^{1}=\text { Cauchy data } f^{2} \text {. }
$$

We denote the Cauchy data by $C D(f)$.

In the present paper we shall be concerned mostly with the situation in which $Y$ is a linear space and the $\Omega^{j}$ are convex sets each containing $Y$ in its boundary. These restrictions are forced upon us because we use Fourier analysis techniques in the spirit of [2] (which will henceforth be referred to as FA).

Most of our considerations will be concerned with the relation (1.3) or with some slight modifications. However our methods would seem to apply to more general situations. Unfortunately our proofs are complete only in case $\operatorname{dim} Y=1$ and the $\mathbf{D}^{j}$ are elliptic, or for a certain compact analog of these problems.

Let us give four interesting examples which illustrate the range of our results.

EXAmple 1. Edge - OF - THE - Wedge TheOREM. Here $n=2 m$ and we have two convex sets $\Omega^{1}$ and $\Omega^{2}$. $\mathbf{D}^{1}=\mathbf{D}^{2}=\mathbf{D}$ is the Cauchy-Riemann system for holomorphic functions of several complex variables, namely,

$$
\frac{\partial f^{j}}{\partial x_{k}}+i \frac{\partial f^{j}}{\partial x_{m+k}}=0, \quad k=1,2, \ldots, m .
$$

$\Omega^{j}$ is a tube over a proper convex cone in the imaginary space; that is, $\Omega^{j}=$ $\left\{x \mid\left(x_{m+1}, \ldots, x_{2 m}\right) \in \Gamma^{j}\right\}$ where $\Gamma^{j}$ is a proper convex cone. Equation (1.2) becomes

$$
f^{1}=f^{2} \quad \text { on } x_{m+1}=x_{m+2}=\cdots=x_{2 m}=0 .
$$

The conclusion is that there is a holomorphic function $f$ on the tube over the convex hull of $\Omega^{1} \cup \Omega^{2}$ whose restriction to $\Omega^{j}$ is $f^{j}$ for all $j$ (see e.g. [9]).

EXAmple 2. Martineau's Edge - OF - THE - Wedge TheOREM. Instead of having two wedges $\Omega^{j}$ we allow ourselves an arbitrary number of them. Equation (1.2) becomes

$$
\sum f^{j}=0 \quad \text { on } x_{m+1}=x_{m+2}=\cdots=x_{2 m}=0 .
$$

A. Martineau proved (see [9]) that there exist functions $g_{i j}$ with $g_{i j}=-g_{j i}$ which are holomorphic on the convex hull of $\Omega^{i} \cup \Omega^{j}$ so that

$$
f^{i}=\sum_{j} g_{i j}
$$

Example 3. Classical Reflection Theorem. If $f$ is harmonic in $x_{n} \geqslant 0$, all $x_{1}, \ldots, x_{n-1}$, and $f$ is $C^{\infty}$ in the closed halfspace, and $f$ or $\partial f / \partial x_{n}$ vanishes on $x_{n}=0$ then $f$ has a harmonic extension to all $R^{n}$. (The condition that $f$ be $C^{\infty}$ in 
the closed half-space can be greatly ameliorated but we shall not be interested in this point here.)

EXAmple 4. H. Lewy's Reflection Theorem. Now $n=2=l$. $\Omega^{1}$ is the upper half-plane and $\Omega^{2}=\Omega^{1}$. $\mathbf{D}^{1}=\Delta$ is the Laplacian and $\mathbf{D}^{2}=\Delta+1$. Equation (1.2) is the equality of Cauchy data, namely equation (1.3). (Here, for simplicity, we assume $f^{j}$ is $C^{\infty}$ in the closed half-plane.) The conclusion is that $f^{j}$ can be extended to a solution of $\mathbf{D}^{j}$ in the whole plane (see [10]).

As far as I know this is the first example of a result of the type we study in which there is more than one $\mathbf{D}^{j}$. Lewy's theorem was reproven by $\mathrm{D}$. Kinderlehrer and $\mathrm{L}$. Nirenberg in [8]. Both of these proofs are restricted to two dimensions. The author's proof was developed at about the same time as that of Kinderlehrer and Nirenberg and was presented in lectures at the Institute for Advanced Study and in Berkeley (see [7]) at that time.

We can actually go further in our investigations of extension theorems. Instead of having one parametrization set $Y$, we can envisage the possibility of having several $Y_{k}$. These are not the $Y^{b}$ involved in the discussions centering around formulas (1.1) and (1.2) but are all of equal dimension and are generally disjoint. We now no longer assume that $Y$ is contained in the closures of all the $\Omega^{j}$ but rather that each $Y_{k}$ is contained in the intersection of some of the $\Omega^{j}$ and there are "chains" of $\Omega^{j}$ joining the various $Y_{k}$. Moreover, for each $k$ there is a fixed parametrization problem of the form (1.1) for all $\Omega^{j}$ whose closures meet $Y_{k}$. Thus we study chain extension and approximation in contrast to star extension and approximation.

By a "chain" joining $Y_{1}$ and $Y_{r}$ is meant a sequence say $\Omega^{1}, \Omega^{2}, \ldots, \Omega^{r+1}$ such that $Y_{j}$ lies in the closure of $\Omega^{j}$, and of $\Omega^{j+1}$. We shall be most concerned with the case $\Omega^{r+1}=\Omega^{1}$.

The chain construction is necessary to treat a very subtle form of Hartogs' extension theorem (see FA for the classical Hartogs' theorem). The classical Hartogs' theorem is concerned with solutions $f$ of $\mathbf{D} f=0$ on $\Omega$ which is, for example of the form $\tilde{\Omega}-K$ where $\tilde{\Omega}$ is an open convex set and $K$ a compact subset. Hartogs' theorem asserts that if $\mathbf{D}$ is truly overdetermined then $f$ extends as a solution of $\mathbf{D}$ over all of $\tilde{\Omega}$.

The proofs given in FA (and all proofs in the literature) depend on the fact that $K$ is compact. We shall present a proof below (for suitable $\mathbf{D}$ ) which allows for some noncompact $K$. We assume that there is only one $\mathbf{D}^{j}$ which we denote by $\mathbf{D}$.

To understand how chains appear, we shall consider the following simple case. Let $V$ be the algebraic variety associated to $\mathbf{D}$ by $\mathrm{FA}$, that is, $V$ is the complex affine algebraic variety of common zeros of the $\hat{D}_{i}$ where $\hat{D}_{i}$ is the Fourier transform of $D_{i}$. Call $l=$ complex $\operatorname{dim} V$ so a parametrization set $Y$ for $\mathbf{D}$ is of real dimension $l$.

Let, then, $Y$ be a linear space of dimension $l$ which is suitable for parametrization. We assume that $l^{\prime}=\operatorname{codim} Y \geqslant 2$; otherwise there is little interest in what follows.

Let $B$ denote the boundary of the unit cube in the space orthogonal to $Y$. Our Hartogs' extension problem is concerned with extensions from a neighborhood of $Y \times B$ over the interior. We define the $Y_{k}$ as the translates of $Y$ to the vertices of $B$. Then the $\Omega^{j}$ are thickenings of suitable faces of $Y \times B$. 
We can modify this construction somewhat. Namely we let $Y^{0}$ be a linear subspace of $Y$. We then call $Y_{k}$ the product $Y_{k}=Y^{0} \times A_{k}$ where the $A_{k}$ are suitable faces of $B$ (which is now the boundary of the unit cube in the space orthogonal to $\left.Y^{0}\right)$ with

$$
\operatorname{dim} A_{k}=\operatorname{dim} Y-\operatorname{dim} Y^{0} .
$$

The $\Omega^{j}$ and the extension problem are as above.

The most extreme case is for $Y^{0}$ a point. The $Y_{k}$ are faces of $B$ of dimension $l$ and the present form of the Hartogs' extension problem is the original (somewhat simplified) case of extension over a compact set. By replacing the cube $B$ by a convex polygon and using the Oka embedding idea of FA we arrive at the formulation of Hartogs' theorem given in FA.

We shall deal only with chains which are formed from the $Y^{0} \times A_{k}$ as above.

The main point of introducing chains is that they allow us to use Fourier analysis to study nonconvex sets.

Our main observation is that "good" star extension implies chain extension. For, the star extension from $Y^{0} \times A_{k}$ gives an extension of a solution $f$ to certain regions. Let $Y^{0} \times A_{k^{\prime}}$ be a vertex adjoining $Y^{0} \times A_{k}$, meaning that there is a region $\Omega^{k k^{\prime}}$ on which $\mathbf{D} f=0$ which contains $Y^{0} \times A_{k}$ and $Y^{0} \times A_{k^{\prime}}$ in its closure. Then we use star extension at $Y^{0} \times A_{k^{\prime}}$ to extend $f$ still further.

Proceeding in this way we use star extension at all the vertices in a chain to extend $f$ to a larger region. We could then partition this new region and continue the process.

Problem. Under what conditions does this method of extension lead to maximal domains of existence for elliptic systems? In particular when does it construct the envelope of holomorphy (for the Cauchy-Riemann system)?

These ideas suggest that there is a "homology theory" for systems D which parallels the topologists' homological theoretic constructions involved in extending cochains from skeletons of a complex. Such a theory would be of great interest but I do not know how to formulate it.

Let us now delve into things with greater precision. We assume that $Y$ is a principal noncharacteristic for all $\mathbf{D}^{j}$ (see FA). This means that the Cauchy problem is well posed for the space of entire functions. It is about the same as the condition

$$
|\hat{t}| \leqslant c(1+|\hat{y}|)
$$

for $(\hat{t}, \hat{y})$ in any $V_{j}$. Here we have written $x=(t, y)$ and $V_{j}$ is the variety of common zeros of the polynomials $\hat{D}_{i}^{j}$.

Moreover, in the case of chains we assume that (after translation to make $A_{k}$ pass through the origin) the linear extension of each $Y^{0} \times A_{k}$ is a principal noncharacteristic for $\mathbf{D}$. (In the case of chains we shall restrict ourselves to only one $\mathbf{D}$.)

In FA we have associated a multiplicity variety to $\mathbf{D}^{j}$ but we shall assume, for simplicity, that this multiplicity variety is just the variety $V_{j}$. This assumption is made for convenience; by complicating our notation we could treat the general case. 
We also assume, for simplicity, that the $\Omega^{j}$ are closed convex sets each containing $Y$ in its boundary so the restriction of solutions to $Y$ has a meaning. In Chapter VI we modify this assumption by combining the present ideas with those of Part I.

Our work will be formulated in the language of FA. We recall some ideas regarding analytically uniform (AU) spaces and localizable analytically uniform (LAU) spaces. A locally convex reflexive topological vector space $W$ is $\mathrm{AU}$ if $W$ is a space of functions or distributions of $x \in R^{n}$ containing all $\exp (i x \cdot \hat{x})$ for $\hat{x} \in C^{n}$ so that $\hat{x} \rightarrow \exp (i x \cdot \hat{x})$ is a holomorphic map of $C^{n} \rightarrow W$. Moreover the linear combinations of $\{\exp (i x \cdot \hat{x})\}$ are dense in $W$. Thus for $S \in W^{\prime}$ the Fourier transform

$$
\hat{S}(\hat{x})=S \cdot \exp (i x \cdot \hat{x})
$$

is an entire function which determines $S$ uniquely. Our final requirement is that the space $\hat{W}^{\prime}$ of $\{\hat{S}\}$ endowed with the topology making the Fourier transform a topological isomorphism can be described intrinsically by seminorms of the form

$$
\|\hat{S}\|_{k}=\sup _{\hat{x} \in C^{n}} \frac{|\hat{S}(\hat{x})|}{k(\hat{x})} .
$$

$\{k\}=K$ is a family of positive continuous functions on $C^{n}$. [ $\infty$ is an allowed value of $k(\hat{x})$.] $K$ is called an AU structure for $W$.

Given any complex affine algebraic variety $V \subset C^{n}$ we can define $\hat{W}^{\prime}(V)$ as the space of entire functions on $V$ which are bounded on $V$ by $c k(\hat{x})$ for any $k \in K$ with the natural topology. An LAU space $W$ has the property that

$$
\left.F \rightarrow F\right|_{V}
$$

is a topological isomorphism of $\hat{W}^{\prime} / I$ onto $\hat{W}^{\prime}(V)$. Here $I$ is the module in $\hat{W}^{\prime}$ generated by the polynomial ideal of $V$.

For each $j$ we pick some LAU space $W_{j}$ of functions or distributions on $\Omega^{j}$. For example, $W_{j}$ could be the space of $C^{\infty}$ functions on $\Omega^{j}$ (recall that $\Omega^{j}$ is closed). Thus we assume that $f^{j}$ of (1.2) belongs to $W^{j}$.

Next we pick some AU space $W$. We want to check if conditions (1.2) imply that each $f^{j} \in W$. As we shall treat the problem in a nonsymmetric fashion we want to know if $f^{1} \in W$.

Naturally, we want to determine the "best" choice for $W$. Within the framework of $\mathrm{AU}$ spaces this is the $\mathrm{AU}$ intersection defined by the relations (1.2). The construction of $\mathrm{AU}$ intersections using relations is given in Chapter VI.

For later purposes we shall give the explicit form of the AU structure of some spaces. For more details see Chapter V of FA.

1. The space $\mathscr{E}=C^{\infty}(R) . \mathscr{E}^{\prime}$ is the space of distributions of compact support. $\hat{\mathscr{E}}^{\prime}$ consists of all entire functions of exponential type which are of polynomial growth on the real axis. Such an $F$ satisfies an inequality of the form

$$
|F(\hat{x})| \leqslant c(1+|\hat{x}|)^{c} \exp (c|\operatorname{Im} \hat{x}|)
$$

for some $c>0$. An AU structure $K=\{k\}$ consists of all continuous positive functions $k(\hat{x})$ which dominate the right side of (1.13) for all $c>0$. 
2. The space $\mathscr{E}_{0}^{+}=C^{\infty}\left(x_{1} \geqslant 0, \ldots, x_{m} \geqslant 0\right)$. Then $\mathscr{E}_{0}^{+\prime}$ consists of all distributions of compact support supported by the closed orthant $\Gamma^{+}: x_{1} \geqslant 0, \ldots, x_{m} \geqslant 0$. $\hat{\mathscr{E}}_{0}^{+\prime}$ consists of all entire functions of exponential type satisfying

$$
F(\hat{x}) \leqslant c(1+|\hat{x}|)^{c} \exp c\left[\max \left(0,-\operatorname{Im} \hat{x}_{1}\right)+\cdots+\max \left(0,-\operatorname{Im} \hat{x}_{m}\right)\right]
$$

The AU structure is defined as before.

A similar result holds for the space $\mathscr{E}^{-}=C^{\infty}\left(\Gamma^{-}\right)$where $\Gamma^{-}=-\Gamma^{+}$. The terms $\max \left(0,-\operatorname{Im} \hat{x}_{j}\right)$ are replaced by $\max \left(0, \operatorname{Im} \hat{x}_{j}\right)$.

3. The space $\mathscr{E}^{+}(\bar{\partial})$ of solutions of the Cauchy-Riemann system on the tube where the imaginary parts $\left(x_{m+1}, \ldots, x_{2 m}\right) \in \Gamma^{+}$.

As above we introduce coordinates $t, y$ where $y=\left(x_{1}, \ldots, x_{m}\right)$ and $t=$ $\left(x_{m+1}, \ldots, x_{2 m}\right)$. The Cauchy surface is $t=0$. By the Fundamental Principle (Theorem 4.1 of FA) the functions and topology of the Fourier transform $\hat{\mathscr{E}}^{+}(\bar{\partial})$ are given by restricting the norms of $\hat{\mathscr{E}}^{+\prime}$ to the Cauchy-Riemann variety $V$. The norms in $\hat{\mathscr{E}}^{+\prime}$ are just the products of the norms in $\hat{\mathscr{E}}_{0}^{+}$(of functions of $\hat{t}$ ) with those of $\hat{\mathscr{E}}^{\prime}$ (of functions of $\hat{y}$ ).

We use $\hat{y}$ as a parameter on $V$. Now $V$ is defined by $\hat{y}_{j}+i \hat{t}_{j}=0$, that is $\hat{t}_{j}=i \hat{y}_{j}$, so that $\operatorname{Re} \hat{y}_{j}=\operatorname{Im} \hat{t}_{j}$. By (1.14) the norms in $\hat{\mathscr{E}}_{0}^{+\prime}$ grow when $\operatorname{Im} \hat{t}_{j} \rightarrow-\infty$ for some $j$, that is, $\operatorname{Re} \hat{y}_{j} \rightarrow-\infty$. This means that we can combine (1.13) and (1.14) to obtain

$$
|F(\hat{y})| \leqslant c(1+|\hat{y}|)^{c} \exp [c(|\operatorname{Im} \hat{y}|+\max (0,-\operatorname{Re} \hat{y}))] .
$$

Here we have written

$$
\max (0,-\operatorname{Re} \hat{y})=\max \left(0,-\operatorname{Re} \hat{y}_{1}\right)+\cdots+\max \left(0,-\operatorname{Re} \hat{y}_{m}\right) .
$$

The AU structure is defined as usual.

The space $\hat{\mathscr{E}}^{-}-(\bar{\partial})$ has similar norms, the only change being the replacement of $\max (0,-\operatorname{Re} \hat{y})$ by $\max (0,+\operatorname{Re} \hat{y})$ in $(1.15)$.

Naturally we could replace the cones $\Gamma^{ \pm}$by arbitrary proper convex cones. These would serve only to complicate our presentation and notation but would add no essential ingredients to our theory.

At this point it is instructive to review the procedures used to obtain various extension theorems. We shall explain why they fail in the cases of star extension and chain extension over a noncompact set. Then we shall introduce a new technique which is the most successful for the problems at hand; this technique is based on the concept of relatively sufficient sets.

The extension methods divide themselves naturally in two categories.

A. Constructions in $W$ or $\hat{W}$.

B. Constructions in $W^{\prime}$ or $\hat{W}^{\prime}$.

Among the former we have

A1. Cohomology method. Suppose we want to extend $f$ from $\Omega$ to $\tilde{\Omega}$ as a solution of $\mathbf{D}$. Then we extend $f$ in some manner to a function $\tilde{f}$ on $\tilde{\Omega}$. Of course, $\mathbf{D} \tilde{f}=\mathbf{g}$ may not vanish, but $\mathbf{g}$ vanishes on $\Omega$. We then must solve a suitable inhomogeneous system, say $\mathbf{D}^{1} h=\mathbf{g}$ with $h$ vanishing on $\Omega$ to correct $\tilde{f}$, that is replace $\tilde{f}$ by $\tilde{f}-h$. 
This method works best when $\tilde{\Omega}-\Omega$ is compact. It is used in FA to prove Hartogs' extension theorem. It does not seem to work for general star or chain extension because $\tilde{\Omega}-\Omega$ is noncompact and we do not know how to use Fourier analysis on $\tilde{\Omega}-\Omega$ to solve the inhomogeneous problem.

A2. Fundamental solutions. We can often use fundamental solutions for $\mathbf{D}$ to extend $f$ from $\Omega$ to $\tilde{\Omega}$. Let $x \in \tilde{\Omega}$ and suppose that we can find a fundamental solution $\mathbf{e}(x)$ for $\mathbf{D}$ with singularity at $x$ [meaning $\sum D_{k} e_{k}(x)=\delta_{x}$ ] so that, for a suitable cutoff function $\chi$ which is 1 near $x$, the support of $\chi \mathbf{e}(x)$ meets the interior of $\Omega$ in a compact set $K(x)$. Then any possible extension $\tilde{f}$ of $f$ must have the value

$$
\tilde{f}(x)=\boldsymbol{\delta}_{x} \cdot \tilde{f}=(\mathbf{D} \cdot \mathbf{e}(x)) \cdot \tilde{f} .
$$

Now,

$$
\mathbf{D} \cdot \chi \mathbf{e}(x)=\delta_{x}+\phi_{x}
$$

where support $\phi_{x} \subset$ interior $\Omega$. Thus (1.16) and (1.17) give

$$
\tilde{f}(x)=(\mathbf{D} \cdot \chi \mathbf{e}(x)) \cdot \tilde{f}-\phi_{x} \cdot \tilde{f}=-\phi_{x} \cdot f
$$

because support $\chi \mathbf{e}(x) \subset$ interior $\Omega$ and $f=\tilde{f}$ on $\Omega$, and also $\mathbf{D} f=0$ by assumption so the first term on the right side of (1.18) vanishes. (Here and in what follows we shall ignore the distinction between $\mathbf{D}$ and $\mathbf{D}^{\prime}$.)

By letting $x$ vary we show that if $\tilde{f}$ is defined to be $-\phi_{x} \cdot f$ then $\mathbf{D} \tilde{f}=0$ and $\tilde{f}$ extends $f$.

This method is used in FA to give another proof of Hartogs' extension theorem. It is also the usual method for proof of the edge-of-the-wedge theorem for holomorphic functions. However, in that case we know many fundamental solutions for D, namely they are some form of $1 / z$ on a holomorphic curve. Thus it is easy to choose an appropriate curve with the proper intersection property.

However I do not know how to use this idea for general $\mathbf{D}$.

Problem. Investigate the use of fundamental solutions for general extension problems.

A3. Fourier representation. We write $f \in W$ in the form

$$
f(x)=\int_{\sigma} e^{i x \cdot \hat{x}} \frac{d \mu(\hat{x})}{k(\hat{x})} .
$$

$\mu$ is a bounded measure (total variation finite) with support contained in a sufficient set $\sigma \subset V$. [A set $\sigma$ is sufficient if the topology of $\hat{W}^{\prime}(V)$ can be defined using norms on $\sigma$ (see FA).] $k$ belongs to an AU structure for $W$. Here $W$ is, for example, the space of $C^{\infty}$ functions on $\Omega$.

By examining (1.19) it is sometimes apparent that $f$ extends to $\tilde{\Omega}$. More generally if $\tilde{W}$ is an LAU space and the norms of $\hat{\tilde{W}}^{\prime}$ on $\sigma$ are not larger than those of $\hat{W}^{\prime}$ then we deduce from (1.19) that $f \in \tilde{W}$.

We think of $f$ being in $\tilde{W}$ as an extension result; it is the usual type of extension result if $\tilde{W}=C^{\infty}(\tilde{\Omega})$.

This method was used extensively in FA. For example it was used to verify hyperbolicity and ellipticity in Chapter VIII. In a slightly different form it can be used to show that certain sequences $\left\{a_{n}\right\}$ are the values $\left\{f^{(n)}(0)\right\}$ for functions $f$ in Denjoy-Carleman classes (Chapter XIII.3). 
It seems that this method is somewhat too weak to apply to the present problems. The difficulty is that $\mu$ or rather $\mu / k$ is not determined by $\sigma$, for $\sigma$ is generally (except when $\operatorname{dim} V=0$ ) not a uniqueness set for Fourier transform. Thus if $f$ is also given to belong to another LAU space $W_{1}$ [this being a simplified version of (1.2)] then we obtain another Fourier representation of $f$, say in terms of $d \mu_{1} / k_{1}$. Even if the support of $\mu_{1} \subset \sigma$ we cannot easily compare $\mu / k$ and $\mu_{1} / k_{1}$ because of the nonuniqueness of representation. For this reason we modify this idea to

A4. Relatively sufficient sets. The main point is that we do not need the representation for $f$ itself but for some representative in $f \bmod \tilde{W}$. Thus if $f$ is in $W$ and in $W_{1}$ then we seek Fourier representations of $f$ as elements of $W / \tilde{W}$ and $W_{1} / \tilde{W}$. The main result of Chapter III of the present work is that under suitable conditions we can construct such representations on a set $\sigma$ which is a uniqueness set for Fourier transform. In practice

$$
\sigma=\{(\hat{t}, \hat{y}) \in V \mid \hat{y} \text { real }\} \text {. }
$$

Another way of formulating this result is that modulo $\tilde{W}$ we are dealing with Fourier analysis which is essentially of classical nature.

Because we are working in quotient spaces, certain obvious constructions become nonobvious. For this reason we must develop an operational calculus in Chapter IV. This will enable us to prove results for star extension in Chapter $\mathrm{V}$ and chain extension in Chapter VI.

In addition to these four extension techniques in $W$ there are techniques in $W^{\prime}$ or $\hat{W}^{\prime}$.

B1. Sufficient sets. This method is dual to A3. To show that $f \in W$ is in $\tilde{W}$ we want to show that if $\tilde{S} \in \tilde{W}^{\prime}$ and $\hat{\tilde{S}}$ (the Fourier transform of $\tilde{S}$ ) is small on $V$ in the topology of $\hat{\tilde{W}}^{\prime}$ then $\hat{\tilde{S}}$ is small on $V$ in the topology of $\hat{W}^{\prime}$. This method works if there is a common sufficient set $\sigma$ for the topologies of $\hat{W}^{\prime}(V)$ and $\hat{\tilde{W}}^{\prime}(V)$ such that the norms of $\hat{\tilde{W}}^{\prime}(V)$ on $\sigma$ are not larger than those of $\hat{W}^{\prime}(V)$.

This method applies essentially in the same cases as A3. It does not work readily when $f$ is given to be in two spaces $W$ and $W_{1}$. For this we have to modify the idea to

B2. Cutting up conditions (See FA pp. 404ff). With $\hat{\tilde{S}}$ as above we try to cut up $\hat{\tilde{S}}$ into

$$
\hat{\tilde{S}}=\hat{S}+\hat{S}_{1}
$$

where $\hat{S} \in \hat{W}^{\prime}(V)$ and $\hat{S}_{1} \in \hat{W}_{1}^{\prime}(V)$ are small in the respective topologies. Thus $f$ defines a continuous linear function on $\tilde{W}^{\prime}$ so is an element of $\tilde{W}$.

We know of three ways to accomplish the cutting up (1.21).

B2a. Cutting up in $C^{n}$. We find appropriate LAU spaces ${ }_{*} \tilde{W},{ }_{*} W$, and ${ }_{*} W_{1}$ whose topologies on $V$ are those of $\tilde{W}, W$, and $W_{1}$ respectively. Moreover we can cut up an ${ }_{*} \tilde{S} \in{ }_{*} \tilde{W}^{\prime}$ in the form

$$
\tilde{*}={ }_{*} S+{ }_{*} S_{1}
$$

in a continuous fashion.

To apply this cutting up in $C^{n}$ we extend (using Theorem 4.1, the Fundamental Principle of FA) $\hat{\tilde{S}}$ from $V$ to an ${ }_{*} \hat{\tilde{S}} \in{ }_{*} \hat{\tilde{W}}^{\prime}$. Then use (1.22) and restrict ${ }_{*} \hat{S}$ and ${ }_{*} \hat{S}_{1}$ to $V$ to obtain $(1.21)$. 
This method is central in Part I.

B2b. Hilbert space. We introduce a Hilbert space structure in $\hat{\tilde{W}}^{\prime}(V)$ (or better in a suitable quotient space) for which the spaces $\hat{W}^{\prime}(V)$ and $\hat{W}_{1}^{\prime}(V)$ are (almost) orthogonal complements. [The expression "almost" is used to take into account relations (1.2).] Then (1.21) is the projection theorem.

This method is being developed at the present time.

B2c. $\bar{\partial}$ cohomology. We make a tentative attempt at (1.21) by writing

$$
\hat{\tilde{S}}=F+F_{1}
$$

where $F=\hat{\tilde{S}}$ when the norms of $\hat{W}^{\prime}(V)$ are larger than those of $\hat{W}_{1}^{\prime}(V)$ and $F_{1}=\hat{\tilde{S}}$ in the converse situation. $F$ and $F_{1}$ are defined in the rest of $V$ so as to be smooth. We now apply the $\bar{\partial}$ operator and attempt to correct $F$ and $F_{1}$ by solving an appropriate $\bar{\partial}$ problem with bounds.

Sometimes this can be solved by an explicit Cauchy formula and sometimes by general $\bar{\partial}$ methods. As in B2b our ideas need further development.

B3. Parametrix method (See FA pp. 407ff). This method is somewhat similar to that of B2 except that we do not have to cut up all $\tilde{S}$ but only suitable "peaking" $\tilde{S}$ as in FA pp. 407ff. Again this technique is in its infancy.

Problem. Develop B2 and B3.

The most successful idea is

B4. Plurisubharmonicity. Suppose we have three AU spaces $W, W_{1}$ and $W_{2}$ and we want to prove that the map $f \rightarrow(f, f)$ of $W$ into $W_{1}+W_{2}$ is surjective on those pairs $\left(f_{1}, f_{2}\right)$ which satisfy the compatibility relations $S_{0} \cdot f_{1}=S_{0} \cdot f_{2}$ when $S_{0} \in$ $W_{1}^{\prime} \cap W_{2}^{\prime}$. By duality we must prove that if $S_{j} \in W_{j}^{\prime}$ and $S_{1}+S_{2} \rightarrow 0$ in the topology of $W^{\prime}$ then we can find $S_{0}$ as above so that $S_{1}-S_{0} \rightarrow 0$ in $W_{1}^{\prime}$ and $S_{2}+S_{0} \rightarrow 0$ in $W_{2}^{\prime}$.

How are we to view this by Fourier transform? The fact that $\hat{S}_{j} \in \hat{W}_{j}^{\prime}$ means that $\hat{S}_{j}$ is not large in certain regions. Let $L_{j}$ be the region where the norms of $\hat{W}_{j}^{\prime}$ are smaller. We seek $\hat{S}_{0}$ which simultaneously approximate $\hat{S}_{1}$ on $L_{1}$ and $-\hat{S}_{2}$ on $L_{2}$ in the respective norms of $\hat{W}_{j}^{\prime}$. Since $\left(\hat{S}_{1}-\hat{S}_{0}\right)+\left(\hat{S}_{2}+\hat{S}_{0}\right) \rightarrow 0$ in $\hat{W}^{\prime}$ this implies that $\hat{S}_{2}+\hat{S}_{0}$ is small in the norms of $\hat{W}^{\prime}$ on $L_{1}$. In general, the norms of $\hat{W}^{\prime}$ and $\hat{W}_{2}^{\prime}$ on $L_{1}$ are about the same since the norms for $\hat{W}_{1}^{\prime}$ are small there. Similarly $\hat{S}_{1}-\hat{S}_{0}$ are small in the norms of $\hat{W}_{1}^{\prime}$ on $L_{2}$.

We can now apply plurisubharmonic estimates. We have estimates for $\hat{S}_{1}-\hat{S}_{0}$ on $L_{1}$ and $L_{2}$ and plurisubharmonicity gives estimates (hopefully) in all of $C^{n}$.

This method is also in its infancy.

We conclude the introduction with a summary of this paper.

Chapter 2 gives a compact analog of all the above. This means that $Y$ is replaced by a torus. The analytic difficulties that appear in the noncompact case disappear and the extension results are easily derived. We also give a detailed treatment of what is meant by a Cauchy problem. Finally we discuss possibilities when the torus group is replaced by other compact groups.

In Chapter 3 we show how to represent solutions of equations as entire solutions plus classical Fourier transforms. This means that, modulo entire functions which play a somewhat trivial role, we are dealing with ordinary Fourier transforms. 
In Chapter 4 we show that certain algebraic operations preserve the congruence modulo entire functions.

Chapters 3 and 4 contain the analysis necessary to pass from the compact theory of Chapter 2 to noncompact $Y$. This is carried out in Chapter 5. Unfortunately we are restricted to $\operatorname{dim} Y=1$ and to elliptic systems.

Chapter 6 contains a dual viewpoint of these problems. Approximations of solutions from the Runge viewpoint are treated. We also consider solutions for which the Cauchy data is defined only in a limit sense as in Part I. We conclude with a formulation of the general extension problem of (1.1) and (1.2) in terms of a new functorial construction on $\mathrm{AU}$ spaces, namely $\mathrm{AU}$ intersection depending on relations (1.2).

2. The compact theory. In many mathematical problems, compactness makes life easier. In the theory of extending solutions of partial differential equations there are two kinds of compactness that play a role. The general Hartogs' theorem which is treated in Chapter XI of FA deals with extension over a compact set. The Paley-Wiener-Schwartz theory enables us to have a good hold on Fourier analysis on compact convex sets and this is the crucial tool in one proof of this Hartogs' theorem.

The other proof of the general Hartogs' theorem of FA is based on the fact that an arbitrary smooth function can be integrated over a compact set.

Hartogs' theorem belongs to the realm of chain extension. We shall now present some ideas concerning star extension where compactness plays a central role.

The compact object is now the Cauchy surface $Y$. In the Introduction we assumed tacitly that $Y$ is a linear space. This assumption was made so that we could apply our usual Fourier transform techniques. These are predicated on

1. Having a good expansion theory of arbitrary functions on $Y$.

2. Being able to describe the solution of the equation whose data has a given expansion.

Even simpler than linear spaces and Fourier integral transform is a torus and Fourier series. Let us illustrate this idea for the compact version of the edge-of-thewedge theorem. We begin by dealing with the Cauchy-Riemann system (1.4). In addition to its intrinsic interest, the compact theory will serve as both a paradigm and a guide to the noncompact theory treated in later chapters.

We use coordinates

$$
r_{k} e^{i \theta_{k}}=x_{k}+i x_{m+k}, \quad k=1, \ldots, m .
$$

The Cauchy-Riemann equations become

$$
e^{i \theta_{k}}\left[\frac{\partial f^{j}}{\partial r_{k}}+\frac{i}{r_{k}} \frac{\partial f^{j}}{\partial \theta_{k}}\right]=0, \quad k=1, \ldots, m .
$$

Here $f^{j}$ is a smooth function on the wedge $\Omega^{j}$ defined by

$$
\left(\log r_{1}, \ldots, \log r_{m}\right) \in \Gamma^{j}
$$

where $\Gamma^{j}$ is a closed proper convex cone. 
The set $Y$ is, of course, the torus $r_{k}=1$ for all $k$. Suppose we have two $j$ values, $j=1,2$. Then the analog of (1.2) is

$$
f^{1}=f^{2} \text { on } Y \text {. }
$$

We assume that $f^{j}$ is smooth in $\Omega^{j}$. The edge-of-the-wedge theorem asserts that there is a function $f$ which is holomorphic when $\left\{\log r_{k}\right\}$ belongs to the convex hull of $\Gamma^{1} \cup \Gamma^{2}$ and restricts to $f^{j}$ on $\Omega^{j}$.

In order to avoid complications of notation, let us suppose that $\Gamma^{1}$ is the positive orthant (i.e. all coordinates positive) and $\Gamma^{2}=-\Gamma^{1}$. We write $f^{ \pm}$in place of $f^{1}, f^{2}$, $\Gamma^{ \pm}$in place of $\Gamma^{j}$, etc. Call $f_{0}^{ \pm}$the restriction of $f^{ \pm}$to $Y$. (Of course, $f_{0}^{+}=f_{0}^{-}$but we shall introduce this later.)

Let us examine the Fourier series of $f_{0}^{ \pm}$. Write

$$
f_{0}^{ \pm}(\theta)=\sum a_{l}^{ \pm} e^{i l \cdot \theta} \text {. }
$$

Then

$$
f^{ \pm}(x)=\sum a_{l}^{ \pm} r^{l} e^{i l \cdot \theta}
$$

with the notation $l=\left(l_{1}, \ldots, l_{m}\right), r^{l}=r_{1}^{l_{1}} \ldots r_{m}^{l_{m}}$, etc.

Now $f^{ \pm}(x)$ is holomorphic in $1<r<\infty$ (resp. $0<r<1$ ). Thus $\left|a_{l}^{ \pm} r^{l}\right|$ is bounded for all $l$ and all of the appropriate $r$. In particular

$$
\left|a_{l}^{ \pm}\right| \leqslant c \exp (-b|l|)
$$

for any $b$ whenever $l$ lies outside of a cone containing the negative (resp. positive) orthant in its interior. Thus "most" of the $a_{l}^{ \pm}$are small and for each $l$ at least one of $a_{l}^{+}, a_{l}^{-}$is small.

We now apply the equality of Cauchy data (2.4) to (2.7) which means that

$$
a_{l}^{+}=a_{l}^{-}
$$

to conclude that (2.7) holds for all $l$. This establishes the compact edge-of-the-wedge theorem.

The true advantage of the compactness of the torus as compared with the noncompactness of the linear space is twofold: In the first place, we have the uniqueness property of Fourier series. This enabled us to go from (2.4) to (2.8). For arbitrary functions on a noncompact linear space our Fourier representation is not unique so we cannot pass from equality of Cauchy data to equality of Fourier representation and this makes the noncompact case much more difficult. In the second place we have absolute convergence of Fourier series of smooth functions. This enabled us to derive (2.7).

Let us apply the above simple method to an edge-of-the-wedge version of $\mathrm{H}$. Lewy's reflection theorem (Example 4 of the Introduction). Let us deal first with the case of two variables.

In polar coordinates the Laplacian is given by

$$
r^{2} \Delta=(r \partial / \partial r)^{2}+\partial^{2} / \partial \theta^{2} .
$$

We assume that we have functions $f^{ \pm}$defined in the regions as before with

$$
r^{2} \Delta f^{+}=\left(r^{2} \Delta-1\right) f^{-}=0 .
$$


The reason we use $r^{2} \Delta$ rather than $\Delta$ as in Example 4 is that $r$ appears in a multiplicative fashion here. The condition (2.4) is replaced by

$$
\begin{aligned}
f^{+} & =f^{-}, \\
\frac{\partial f^{+}}{\partial r} & =\frac{\partial f^{-}}{\partial r} \quad \text { on } Y .
\end{aligned}
$$

Let the Fourier series of $f^{ \pm}$on $Y$ be written as in (2.5) and let the Fourier series of the restrictions of $\partial f^{ \pm} / \partial r$ to $Y$ be given by

$$
\frac{\partial f^{ \pm}}{\partial r}=\sum b_{l}^{ \pm} e^{i l \theta} \quad \text { on } Y .
$$

If we write

$$
f^{ \pm}(r, \theta)=\sum c_{k l}^{ \pm} r^{k} e^{i l \theta}
$$

then (2.10) gives

$$
\begin{aligned}
& k= \pm l \text { for } f^{+}, \\
& k= \pm \sqrt{l^{2}+1} \text { for } f^{-} .
\end{aligned}
$$

We shall write $c \pm \pm$ to denote the four possibilities.

Now apply (2.5) and (2.12). We find

$$
\begin{aligned}
& a_{l}^{ \pm}=c_{+l}^{ \pm}+c_{-l}^{ \pm}, \\
& b_{l}^{+}=l c_{+l}^{+}-l c_{-l}^{+}, \\
& b_{l}^{-}=+\sqrt{l^{2}+1} c_{+l}^{-}-\sqrt{l^{2}+1} c_{-l}^{-} .
\end{aligned}
$$

Equation (2.11) asserts

$$
a_{l}^{+}=a_{l}^{-}, \quad b_{l}^{+}=b_{l}^{-} .
$$

This gives two equations for the four quantities $a_{l}^{ \pm}, b_{l}^{ \pm}$.

As we learned from the compact edge-of-the-wedge theorem we need some inequalities to combine with these equalities. The inequalities come from the fact that $f^{ \pm}$is defined and regular in $\Omega^{ \pm}$. This means that the Fourier coefficients are bounded. This result is a good inequality when $r^{k}$ is large, i.e. in $\Omega^{+}$for $k>0$ and $\Omega^{-}$for $k<0$.

The above thus gives

$$
\begin{aligned}
& c_{+l}^{+}, c_{-l}^{-} \text {exp decreasing for } l>0, \\
& c_{+l}^{-}, c_{-l}^{+} \text {exp decreasing for } l<0 .
\end{aligned}
$$

We are interested in proving that certain quantities are exponentially decreasing. Thus we might as well replace exponentially decreasing by 0 , that is, compute modulo exp decreasing. Then we can write (2.16) and (2.15) as (writing $\equiv$ to mean congruent mod exp decreasing)

$$
c_{-l}^{+} \equiv c_{+l}^{-}, \quad-l c_{-l}^{+} \equiv \sqrt{l^{2}-1} c_{+l}^{-}
$$

for $l>0$ with similar equations for $l<0$. It is clear that multiplication by $l$ or $\sqrt{l^{2}+1}$ preserves congruence. Thus

$$
\left(l+\sqrt{l^{2}-1}\right) c_{+l}^{-} \equiv 0 .
$$


Since the coefficient $l+\sqrt{l^{2}+1}$ does not approach zero rapidly (in fact it is bounded from below) we conclude that $c_{+l}^{-} \equiv 0$. By (2.18) it follows that $c_{ \pm}^{ \pm}$are all $\equiv 0$ for $l>0$. A similar result (using the fact that $\sqrt{l^{2}+1}-l$ is not too small at infinity) holds for $l<0$ so we have the desired result.

Let us now pass to higher dimensions.

Our first candidate for a high dimension Lewy problem is to have $f^{+}$pluriharmonic in $\Omega^{+}$while $f^{-}$satisfies a perturbed pluriharmonic system. We write

$$
\frac{\partial}{\partial \bar{z}_{s}}=\frac{\partial}{\partial x_{s}}+i \frac{\partial}{\partial x_{m+s}}=e^{i \theta_{s}}\left(\frac{\partial}{\partial r_{s}}+\frac{i}{r_{s}} \frac{\partial}{\partial \theta_{s}}\right) \text {. }
$$

The equations are

$$
\frac{\partial^{2} f^{+}}{\partial z_{s} \partial \bar{z}_{t}}=0, \quad\left(z_{s} \bar{z}_{t} \frac{\partial^{2}}{\partial z_{s} \partial \bar{z}_{t}}-\alpha_{s t}\right) f^{-}=0
$$

for constants $\alpha_{s t}$. [We could have also perturbed the first equation in (2.21) by constants but that would have no effect on our method.]

The equality of Cauchy data is

$$
f^{+}=f^{-}, \quad \frac{\partial f^{+}}{\partial r_{s_{0}}}=\frac{\partial f^{-}}{\partial r_{s_{0}}} \quad \text { on } Y \text { for some } s_{0} .
$$

(This point will be clarified below.) We shall simply set $s_{0}=1$. We also use the same notations (2.5) and (2.12) for the Fourier series on $Y$ of $f^{ \pm}$and $\partial f^{ \pm} / \partial r_{1}$ [except that now $\left.l=\left(l_{1}, \ldots, l_{m}\right)\right]$. We also write $f^{ \pm}(r, \theta)$ in the form (2.13).

Using (2.20) we have

$$
\left(z_{s} \bar{z}_{t} \frac{\partial^{2}}{\partial z_{s} \partial \bar{z}_{t}}-\alpha_{s t}\right) f^{-}=\sum\left[\left(k_{s}+l_{s}\right)\left(k_{t}-l_{t}\right)-\alpha_{s t}\right] c_{k l}^{-} r^{k} e^{i l \theta} .
$$

Thus $k$ is determined from $l$ by the equations

$$
\left(k_{s}+l_{s}\right)\left(k_{t}-l_{t}\right)-\alpha_{s t}=0 \text { all } s, t .
$$

Unfortunately, (2.24) has few solutions. This is easily seen as follows. By setting $s=t$ we see that for large $\left|l_{s}\right|$ we have $k_{s} \sim \pm l_{s}$. If e.g. $k_{s} \sim+l_{s}$ then (2.24) cannot hold for all $l_{s}$ for fixed $k_{t}, l_{t}$ unless $\alpha_{s t}=0$ and $k_{t}=l_{t}$.

Hence if we want solutions of (2.24) for all $l$ we must have all $\alpha_{s t}=0$. This case is treated just as the ordinary compact edge-of-the-wedge treated above.

It seems that a more interesting situation is arrived at by considering (2.21) for $s=t$ only. Then (2.22) takes the form

$$
\begin{aligned}
f^{+} & =f^{-}, \\
\frac{\partial^{p} f^{+}}{\partial r_{p_{1}} \cdots \partial r_{p_{p}}} & =\frac{\partial^{p} f^{-}}{\partial r_{p_{1}} \cdots \partial r_{p_{p}}} \quad \text { on } Y
\end{aligned}
$$

for all $p_{1}<p_{2}<\cdots<p_{p}$. We use the same notation as before. (2.24) is required only for $s=t$ so for each $l$ there are $2^{m}$ solutions which coincides with the number of Cauchy data given in (2.25). The complete understanding of the meaning of the Cauchy problem (2.25) is found in our work [4]. We shall say a little more about it below. 
Let us examine the analogs of equation (2.14). These are

$$
\begin{aligned}
& k_{s}= \pm l_{s} \text { for } f^{+}, \\
& k_{s}= \pm \sqrt{l_{s}^{2}+\alpha_{s}} \text { for } f^{-} .
\end{aligned}
$$

We assume for simplicity that $\alpha_{s}>0$. (We have written $\alpha_{s}$ for $\alpha_{s s}$.)

To formulate the analog of (2.15) let us call $\left\{h_{j}\right\}$ the polynomials $k_{p_{1}} \cdots k_{p_{p}}$ for any $p_{1}<p_{2}<\cdots<p_{p}$. (We allow $p=0$ in which case we obtain the empty product which is 1.) We denote by $\partial\left(h_{j}\right)$ the differential operator of $(2.25)$. Instead of using the notation $a_{l}^{ \pm}, b_{l}^{ \pm}$as in (2.5) and (2.12) we use the notation $c\left(h_{j}\right)\left(f^{ \pm}\right)_{l}$ to denote the Fourier series coefficients of $\partial\left(h_{j}\right) f^{ \pm}$on $Y$. (2.15) can now be written in the form

$$
c\left(h_{j}\right)\left(f^{ \pm}\right)_{l}=\sum h_{j}(k) c_{k l}^{ \pm} .
$$

The sum is over all $k$ which "lie above $l$ " meaning that they are defined by (2.26).

Next we need the analogs of equations (2.17). Where do we have exponential decrease of the $c_{k l}^{ \pm}$? As usual this occurs for $c_{k l}^{ \pm}$when $k$ lies outside a cone $\tilde{\Gamma}^{\mp}$ containing the negative (resp. positive) orthant in its interior. For any sufficiently large $l$ there can be at most $2^{m-1}$ values of $k$ for either $f^{+}$or $f^{-}$which lie in $\tilde{\Gamma}^{-}$or in $\tilde{\Gamma}^{+}$respectively since the components of $k$ are, up to sign, close to those of $l$. Thus many lie in $\tilde{\Gamma}^{\mp}$ if many components of $l$ are small. For fixed $l$ we denote by $k^{ \pm}$those $k$ corresponding to $l$ by (2.26) which lie in $\tilde{\Gamma}^{\mp}$.

The equality of Cauchy data of $f^{ \pm}$takes the form

$$
\sum h_{j}\left(k^{+}\right) c_{k^{+} l}^{+} \equiv \sum h_{j}\left(k^{-}\right) c_{k^{-} l}^{-}
$$

the sums being respectively over the at most $2^{m-1}$ values of $k^{ \pm}$. (2.28) is the general form of (2.18). It is a system of $2^{m}$ equations involving at most $2^{m}$ quantities $c_{k^{ \pm}}^{ \pm} l$. Our general edge-of-the-wedge theorem means that the rank of the matrix $\left\{h_{j}\left(k^{ \pm}\right)\right\}$ is maximal, that is, equal to the number of $k^{ \pm}$and, moreover, there is a submatrix of maximal rank whose determinant is not too small.

Rather than go into the algebra involved in establishing this result, let us first put the above construction in a general setting. This is provided us by our work [4] on harmonic functions; we shall extract some ideas needed for the present situation and later chapters.

Let $i_{1}, \ldots, i_{q}$ (with $q \leqslant m$ ) be homogeneous polynomials in $k$ of positive degree. We assume that the $i_{t}$ are strongly independent which means that the complex varieties $i_{1}=c_{1}, \ldots, i_{q}=c_{q}$ for $c_{1}, \ldots, c_{q}$ arbitrary constants always have codimension exactly $q$. By $\partial\left(i_{t}\right)$ we denote the differential operator obtained from $i_{t}$ by replacing $k_{s}$ by $\partial / \partial k_{s}$. A function $h$ is called harmonic if

$$
\partial\left(i_{t}\right) h=0 \text { all } t .
$$

The important property of harmonic functions is that the homogeneous harmonics give a basis for suitable functions on varieties of the form $i_{1}=c_{1}, \ldots, i_{q}=c_{q}$ even when $c_{t}$ are polynomials with degree $c_{t}<$ degree $i_{t}$. 
To make contact with the edge-of-the-wedge theorem, we introduce a new variable $l$. We next consider varieties in $k, l$ variables

$$
V=\left\{(k, l) \mid i_{t}(k)=j_{t}(l)\right\}
$$

where degree $j_{t}=$ degree $i_{t}$. More generally, we consider varieties defined by equations of the form

$$
i_{t}(k)+Q_{t}(k, l)=0
$$

where degree $Q_{t}=$ degree $i_{t}$ and the degree of $Q_{t}$ in $k$ is $<$ degree $i_{t}$. Suitable functions on $V$ are parametrized by the restrictions to $V$ of functions of the form

$$
\sum g_{u}(l) h_{u}(k) \text {. }
$$

We may say that suitable function spaces on $V$ are tensor products of function spaces in $l$ with the space of harmonics.

In our case the polynomials $i_{s}=k_{s}^{2}$. The harmonics are spanned exactly by $k_{p_{1}} k_{p_{2}} \cdots k_{p_{p}}$ with $p_{1}<p_{2}<\cdots<p_{p}$. In our case we have two varieties $V^{ \pm}$ where $V^{+}$is defined by $k_{s}^{2}=l_{s}^{2}$ and $V^{-}$by $k_{s}^{2}=l_{s}^{2}+\alpha_{s}$. Of course, in the present chapter we are considering $l$ to vary over lattice points but that does not effect the above formalism seriously.

We are now in a position to understand the general setting of the Lewy edge-of-the-wedge theorem. Suppose we are given two elliptic systems $\mathbf{D}^{+}, \mathbf{D}^{-}$of equations in $r, \theta$. [In the present situation these systems involve polynomials in $r_{s} \partial / \partial r_{s}$ and $\partial / \partial \theta_{s}$.] Thus $\mathbf{D}^{ \pm}$correspond to algebraic varieties $V^{ \pm}$in $(k, l)$ space where $l$ is a lattice variable. We assume that the $\theta$ torus is a Cauchy surface for both $\mathbf{D}^{ \pm}$and that the Cauchy data is the same for both $\mathbf{D}^{ \pm}$. In terms of our above description of harmonic functions this means that we can use the same harmonic functions $\left\{h_{r}(k)\right\}$ to parametrize both the varieties $V^{ \pm}$over each $l$.

As before, suppose $f^{ \pm}$is a solution of $\mathbf{D}^{ \pm}$in $\Omega^{ \pm}$. (We do not need all of $\Omega^{ \pm}$; a similar theory would hold if $\mathbf{D}^{ \pm} f^{ \pm}=0$ on a fixed neighborhood of $Y$ in $\Omega^{ \pm}$; this point will be discussed later. At present we shall work with the somewhat simpler case that the solutions are on all of $\Omega^{ \pm}$.) As we have seen before, the "bad region" that is the region on which we have no good estimates on the Fourier coefficients $c_{k l}^{ \pm}$ of $f^{ \pm}$occurs when $\operatorname{Re} k$ lies in a neighborhood of $\hat{\Gamma}^{ \pm}$which is the dual cone (in $k$ space) to $\Gamma^{ \pm}$.

Now, the systems $\mathbf{D}^{ \pm}$are assumed to be elliptic. Thus for large $|l|$ the $k$ coordinates of the points $(k, l) \in V^{ \pm}$have large real parts. In fact, by the results of Chapter VIII of FA we have

$$
|\operatorname{Re} k| \geqslant c|l|
$$

for $l$ large. This means that we can ignore all $c_{k l}^{ \pm}$except those for which $\operatorname{Re} k$ is as above, that is,

$$
f^{ \pm}(r, \theta) \equiv \sum_{\operatorname{Re} k \text { near } \hat{\Gamma}^{ \pm}} c_{k l}^{ \pm} r^{k} e^{i l \theta}
$$

because the other $c_{k l}^{ \pm}$are exponentially decreasing. 
Let us denote by $q$ the generic number of points $k$ for which $(k, l) \in V^{ \pm}$. Thus $q$ is the number of Cauchy data. Now, for any $l$ we have some of the corresponding $\operatorname{Re} k$ in $\hat{\Gamma}^{+}$and some others in $\hat{\Gamma}^{-}$. Suppose first that $V^{+}=V^{-}$. We can arrange the neighborhoods in (2.33) so no $k$ belongs to both neighborhoods, at least for $|l|$ large. The equality of Cauchy data of $f^{+}$and $f^{-}$can be written in the form

$$
\sum_{\operatorname{Re} k \text { near } \hat{\Gamma}^{+}} c_{k l}^{+} h_{s}(k) e^{i l \theta} \equiv \sum_{\operatorname{Re} k \text { near } \hat{\Gamma}^{-}} c_{k l}^{-} h_{s}(k) e^{i l \theta}
$$

for each $s$. Since the $k$ occurring in both sums all lie over $l$ and no $k$ occurs in both sums, if we had equality in (2.34) instead of congruence, we could conclude that $c_{k l}^{ \pm}=0$ for all $k, l$ because of the uniqueness property of harmonic expansion. (Although in this special case we could, by a simple device, replace congruence by equality, we shall not do it with a view to deeper applications.)

Thus we need the following

Proposition 2.1. Suppose that $V$ has no real multiple characteristics, that is, the $k$ above real $l$ are distinct at infinity. If

$$
\left|\sum_{(k, l) \in V} c_{k} h_{s}(k)\right| \leqslant 1
$$

then for l large

$$
\left|c_{k}\right| \leqslant \alpha(1+|l|)^{\alpha}
$$

for some $\alpha$.

Proof. Call $b_{s}=b_{s}(l)$ the sum in (2.35). We want to invert the matrix $\left\{h_{s}(k)\right\}$. We know that it is generically invertible so that its determinant $d=\operatorname{det} h_{s}(k)$ is a function of $l$ which does not vanish identically. Moreover, $d$ is an algebraic function of $l$ since the $h_{s}$ are polynomials.

Now, $d$ can vanish only for those $l$ for which there is a coincidence of $k$ above $l$ since when the $k$ are distinct the $h_{s}(k)$ give a basis for functions on that set of $k$ (see [4] for details). Thus $d$ is an algebraic function which does not vanish near the real points at infinity in $l$ space. This implies (see Chapter I of FA) that $|d(l)| \geqslant|l|^{-u}$ for some $u$ for $l$ large.

Since the $h_{s}$ are polynomials they are bounded by a power of $|l|$. Proposition 2.1 is thereby proven.

Next let us pass to the case of $V^{+}$not necessarily equal to $V^{-}$. Suppose also that for any $l$ the number of $k$ in $V^{+}$with $\operatorname{Re} k$ near $\hat{\Gamma}^{+}$plus the number of $k$ in $V^{-}$ with $\operatorname{Re} k$ near $\hat{\Gamma}^{-}$is $\leqslant q$. This will be the case when $V^{ \pm}$are asymptotically the same as in the case in Lewy's theorem (Example 4 of Chapter I).

Problem 2.1. Find algebraic conditions to allow the conclusion (2.36) of Proposition 2.1 when the sum in (2.35) is taken over $k$ in $V^{+}$with $\operatorname{Re} k$ near $\hat{\Gamma}^{+}$and also $k$ in $V^{-}$with $\operatorname{Re} k$ near $\hat{\Gamma}^{-}$.

CONJecture. Asymptotic equality of $V^{ \pm}$, that is, the identity of points at infinity of $V^{ \pm}$over real $l$, is a sufficient condition for (2.36) to hold. 
The above construction leads to

THEOREM 2.2. When $V^{ \pm}$have no real multiple characteristics and when $V^{+}=V^{-}$or when the algebraic conditions required by Problems 2.1 are satisfied then for elliptic $\mathbf{D}^{ \pm}$the edge-of-the-wedge theorem holds, that is, $f^{ \pm}$extend to solutions of $\mathbf{D}^{ \pm}$ respectively in the whole space except where some $n_{j}=0$.

REMARK 1. If we consider $V=V^{+} \cup V^{-}$then we should usually have $2 q$ points in $V$ above a generic $l$. For $V$ we should give $2 q$ Cauchy data, say defined by harmonics $\tilde{h}_{s}$. Now, $f^{ \pm}$are both solutions of the equations corresponding to $V$. Thus if the Cauchy data using $\left\{\tilde{h}_{s}\right\}$ of $f^{+}$and $f^{-}$coincide then Proposition 2.1 applies so Theorem 2.2 says that $f^{ \pm}$extend to solutions in the whole space of the system $\mathbf{D}$ corresponding to $V$.

Thus $2 q$ Cauchy conditions suffice for the extendibility of $f^{ \pm}$to a solution of $\mathbf{D}$. But Theorem 2.2 allows for the reduction from $2 q$ to $q$ and to extension to solutions of $\mathbf{D}^{ \pm}$.

EXAMPLE. We give a simple example for which we cannot reduce the number of conditions below $2 q$. This is $V^{ \pm}=\{(k, l) \mid k= \pm i l\}$. It is readily seen that two Cauchy conditions are needed. Of course, in this case $V^{ \pm}$are far from being asymptotically equal.

REMARK 2. Although we do not know how to solve Problem 2.1 in general we can successfully treat the case (2.25) ff. We can show that for generic $\left\{\alpha_{s}\right\}$ that is, except for an algebraic variety (which is a sort of eigenvalue variety) that the solution to Problem 2.1 is in the affirmative so the conclusion of Theorem 2.2 holds. We shall omit the complicated proof as we are hoping to find a more elegant one.

REMARK 3. In case $V^{+}=V^{-}$we can eliminate the hypothesis that $\mathbf{D}$ be elliptic. This is treated in Theorem 2.5.

The previous discussion has centered around the edge-of-the-wedge theorem. Let us explain the modifications necessary to treat a general classical reflection theorem (see Example 3 of Chapter I).

In this case we deal with only one function, say $f^{+}$defined on the tube over $\Gamma^{+}$as before. Instead of edge-of-the-wedge conditions (2.34) we have reflection conditions

$$
\sum_{\operatorname{Re} k \text { near } \hat{\Gamma}^{+}} c_{k l}^{+} h_{s}(k) e^{i l \theta} \equiv 0 .
$$

Instead of imposing (2.37) for all $s$, we need only $q^{+}$conditions where $q^{+}$is the maximal number of $k$ at infinity in the sum in (2.37).

The problem we face is that of choosing $q^{+}$of the $h_{s}$ so that the matrix $\left(h_{s}(k)\right)$ is generically of maximal rank, or, equivalently, these $h_{s}$ span the functions at the $k$ in (2.37) lying over a generic $l$. In any given example it is easy to find good choices of the $h_{s}$ but we do not know how to solve the general

Problem 2.2. Determine for which $q^{+}$of the $h_{s}$ the matrix $\left(h_{s}(k)\right)$ is generically of maximal rank.

Our above ideas yield (D is not assumed to be elliptic). 
THEOREM 2.3. Any set of $q^{+}$of the $h_{s}$ satisfying the conditions of Problem 2.2 gives a reflection theorem in the sense that if the corresponding Cauchy data of $f^{+}$vanish on $Y$ then $f^{+}$extends to a solution $\mathbf{D}^{+}$in the whole space except where some $r_{j}=0$.

REMARK 1. Problems 2.1 and 2.2 have a certain opposite flavor. For Problem 2.1 is concerned with using the $h_{s}$ to parametrize sets more general than those above $l$ in $V^{ \pm}$while Problem 2.2 is concerned with finding subsets of the $h_{s}$ to parametrize subsets of points above $l$ in $V^{+}$.

REMARK 2. We can, of course, combine the ideas of Theorems 2.2 and 2.3 to prove an extension result when some Cauchy data of $f^{ \pm}$vanish and others are equated.

We give now a brief indication of how to replace the cones $\Gamma^{ \pm}$by more general convex cones which we denote by $\Gamma^{1}, \Gamma^{2}$. The dual cones are $\hat{\Gamma}^{1}, \hat{\Gamma}^{2}$. We assume that $\mathbf{D}^{1}=\mathbf{D}^{2}=\mathbf{D}$ but we do not assume that $\mathbf{D}$ is elliptic.

In order to understand the situation properly, we rewrite (2.34) in the form

$$
\left(c_{k l}^{1}-c_{k l}^{2}\right) h_{s}(k) e^{i l \theta}=0
$$

for all $s$. Equation (2.38) differs from (2.34) only in that we put into (2.38) the terms which are small at infinity. (2.38) implies

$$
c_{k l}^{1}=c_{k l}^{2}
$$

Of course (2.39) is useful when we know that one of the $c_{k l}^{j}$ is small, which is the situation in (2.34) because $\hat{\Gamma}^{+}$and $\hat{\Gamma}^{-}$are far apart.

Proposition 2.4. The dual of the convex hull of $\Gamma^{1} \cup \Gamma^{2}$ is $\hat{\Gamma}^{1} \cap \hat{\Gamma}^{2}$.

Proof. Presumably this proposition is well known but we give a proof since we do not know any reference.

Clearly a vector $\hat{t}$ is positive on the convex hull of $\Gamma^{1} \cup \Gamma^{2}$ if and only if $\hat{t}$ is positive on both $\Gamma^{1}$ and $\Gamma^{2}$. This is the same as saying $\hat{t} \in \hat{\Gamma}^{1} \cap \hat{\Gamma}^{2}$ which is the result.

Thus for those $k$ which lie in $\hat{\Gamma}^{1} \cap \hat{\Gamma}^{2}$ the equation (2.39) gives no new inequality on the $c_{k l}^{j}$. On the other hand, for $k \notin \hat{\Gamma}^{1} \cap \hat{\Gamma}^{2}$ at least one of $c_{k l}^{j}$ is small so (2.39) implies that the other one is small. Here $c_{k l}^{j^{\prime}}$ being small means that $\left|c_{k l}^{j} r^{k}\right|$ is bounded for $\log r \notin \Gamma^{j}$. For $k$ lying outside both $\hat{\Gamma}^{1}$ and $\hat{\Gamma}^{2}$ the quantity $\left|c_{k l}^{j} r^{k}\right|$ is bounded when $\log r \notin \Gamma^{1} \cup \Gamma^{2}$. We find easily (see Chapter V for more details) that thus we can write

$$
f^{j}(r, \theta) \equiv \sum_{k \text { near } \hat{\Gamma}^{1} \cap \hat{\Gamma}^{2}} c_{k l}^{j} r^{k} e^{i l \theta}
$$

The series in (2.40) converges for $\log r$ in any proper subcone $\Gamma$ of the convex hull of $\Gamma^{1} \cup \Gamma^{2}$.

We have shown

THEOREM 2.5. Let $\mathbf{D}^{1}=\mathbf{D}^{2}$ and suppose $\mathbf{D}^{j} f^{j}=0$ when $\log r \in \Gamma^{j}$. If the Cauchy data of $f^{j}$ agree on $Y$ then $f^{j}$ extend to a solution $\tilde{f}^{j}$ for $\log r$ in the convex hull of $\Gamma^{1} \cup \Gamma^{2}$. 
REMARK 1. I do not know of a good analog of Theorem 2.5 when $\mathbf{D}^{1} \neq \mathbf{D}^{2}$.

REMARK 2. In this compact case the result is true without any ellipticity assumption. (The proof does not use ellipticity.) However the noncompact analog given in Chapter $\mathrm{V}$ requires ellipticity.

Thus far we have discussed only two systems $\mathbf{D}^{ \pm}$and the relation between solutions $f^{ \pm}$given by equality of the Cauchy data. We may envisage having several systems $\mathbf{D}^{j}$ and corresponding solutions $f^{j}$ in sets where $\log r$ lies in suitable convex cones $\Gamma^{j}$. We still assume that we have a single harmonic system $\left\{h_{s}\right\}$ that defines a Cauchy problem with data on the torus $Y$ for all the $\mathbf{D}^{j}$.

We suppose all the $\mathbf{D}^{j}$ are elliptic with distinct real characteristics. Then relation (2.34) is replaced by

$$
\sum_{s j} e_{t j}^{s} \sum_{k} c_{k l}^{j} h_{s}(k) \equiv 0
$$

for $t=1, \ldots, q_{1}$. The sum is over only those $k$ for which $(k, l) \in V^{j}$ and $c_{k l}^{j} \not \equiv 0$ as above.

The simplest analog of the above case of two values of $j$ occurs when the number $\tilde{q}$ of $k$ in the sum (2.41) for large $l$ for all $j$ is $\leqslant q$. Then we are in a situation close to that of Problem 2.1 if the coefficients $e_{t j}^{s}$ satisfy reasonable conditions.

In case the number of $k$ is larger than $q$ then we could increase the number of $q_{1}$ as in the above Remark 1. A second possibility is to derive all the growth information we can from (2.41).

What sort of conclusions can we expect? The analog of (2.8) which is relevant to Martineau's edge-of-the-wedge theorem discussed in Chapter 1 is of the form

$$
c_{i}^{1}+c_{i}^{2}+c_{i}^{3} \equiv 0 \text {. }
$$

The conclusion from this is that the largest of the $c_{i}^{j}$ cannot be larger than the second largest except for an unimportant constant which we shall ignore.

In general we expect that (2.41) should imply the largest $q_{1}$ of the $c_{k l}^{j}$ cannot be larger than the $\left(\tilde{q}-q_{1}\right)$ th largest. (In particular when $\tilde{q}=q_{1}$ then all the $c_{k l}^{j}$ are $\equiv 0$ as we mentioned above.)

Problem 2.3. Generalize Problem 2.1 so as to find conditions in which we can assert that the largest $q_{1}$ of the $c_{k l}^{j}$ cannot be larger than the $\left(\tilde{q}-q_{1}\right)$ th largest.

The algebra in case of Martineau's edge-of-the-wedge theorem is simple and we leave it as an exercise.

Our treatment of the edge-of-the-wedge theorem yields results for chain extension. We now consider solutions $f$ of a single system $\mathbf{D} f=0$ which are defined near the boundary of a cube in $r$ times all of $Y$. Actually we need only that $f$ be defined near the one skeleton of this cube times $Y$.

Each vertex $v$ of the cube is an edge for the edge-of-the-wedge theorem for $\mathbf{D}$ where the wedges are neighborhoods of one dimensional faces $a_{j}^{v}$ which form the one star of $v$. We assume that the Cauchy data of $f$ regarded as a solution on $a_{j}^{v} \times Y$ all agree at $v \times Y$. Then the equality in (2.38) for any pair of $j$ implies, as in the proof of Proposition 2.4 that the function $f$ extends to a solution on the convex hull of the one star of $v$ in the two skeleton times $Y$. 
We can iterate this argument, passing from the two skeleton to the three skeleton, etc. We obtain

THEOREM 2.6. Let $\mathbf{D}$ be an elliptic system for which $Y$ is a Cauchy surface. Then any solution of $\mathbf{D} f=0$ defined near the one skeleton of a cube in $r$ times all $Y$ extends to a solution defined on the neighborhood of the full cube times $Y$.

REMARK. $f$ does not have to be defined in a full neighborhood of the vertices (times $Y$ ) but only, near each vertex, in a union of cones containing the one star of the vertex. Of course, the Cauchy data corresponding to the various edges emanating from a fixed vertex must be equal. The conclusion is that $f$ extends to a solution on the interior of the cube times $Y$.

It is possible to reformulate the Lewy edge-of-the-wedge idea so as to put it in a new setting. Consider the polynomials $i_{s}(x)=x_{s}^{2}+x_{s+m}^{2}$. Then the torus $Y$ can be described as $i_{s}=1$ for all $s$. Our general theory asserts that the harmonics defined by any strongly independent polynomials as above give a basis for functions on $Y$. A function $h$ is harmonic if it satisfies $\Delta_{s} h=0$ for all $s$. If we define a basis for harmonics using homogeneity then we obtain $\left\{r^{|l|} \exp (i l \theta)\right\}$. Thus the restrictions to $Y$ yield the Fourier series basis on $Y$.

The above description depends on the fact that we are dealing with functions $h$ which are harmonic in the whole space. The other harmonics which played a crucial role in the edge-of-the-wedge theorem have singularities where some of the $r_{s}=0$.

Similarly, for the "deformed" system $\left\{r_{s}^{t} \Delta_{s}-\alpha_{s}\right\}$ we must analyse solutions which take the value $\exp (i l \theta)$ on $Y$.

We can thus formulate the above procedure in the following steps.

(1) Use harmonicity in all $x$ space to obtain a basis like $\exp ($ il $\theta)$ for functions on a set $Y$ of the form $i_{t}=c_{t}$.

(2) Study functions which are harmonic near $Y$. The description of these necessitates a Cauchy like problem on $Y$ since for each basis element $\phi$ there are several harmonics near $Y$ which restrict to $\phi$ on $Y$. This Cauchy problem may be related to another harmonicity theory in new variables, say $k, l$.

(3) Do the same for a perturbed system. (We must find perturbations which fit into the harmonic theory.)

(4) Split the Cauchy data [for the Cauchy problem of (2)] between the perturbed systems. Is there still a good harmonicity theory as in Problem 2.1?

We feel that this program could be carried out to a reasonable extent in case the $i_{t}$ are the invariants of a nice compact group such as the rotation group.

Problem 2.4. Carry out this program in the group case.

3. Relatively sufficient sets. As mentioned in the introduction we shall prove theorems primarily using $\hat{W}$ rather than $\hat{W}^{\prime}$. The reason is that in our problem we are really working modulo the $\mathrm{AU}$ intersection. Since the $\mathrm{AU}$ intersection is generally dense in all the $W_{j}$ we cannot make much sense of the dual of $W_{1} / \bigcap^{\mathrm{a}} W_{j}$. Nevertheless the proofs generally depend heavily on the dual space. 
The simplest result in this direction is

TheOREM 3.1. Any $C^{\infty}$ function $f$ on all of $R^{n}$ admits a decomposition

$$
f=h+g
$$

where $h$ is entire and $g$ is a classical Fourier transform meaning

$$
g(x)=\int e^{i x \cdot \hat{x}} \frac{d \hat{\mu}(\hat{x})}{k(\hat{x})}
$$

where the integral is over $R^{n}$. Here $\mu$ is a bounded measure and $k$ dominates all polynomials.

It should be remarked that the case $n=1$ is found in [6]. However the method of that paper does not apply to $n>1$ without some modification.

REMARK 1. After writing this theorem and its proof I discovered a note by P. M. Gauthier in Proceedings of approximation theory, St. John's, 1984 in which two proofs of a result which is essentially the same as Theorem 3.1 are given.

REMARK 2. We can interpret Theorem 3.1 as the assertion that the real axis is sufficient for $\mathscr{E}\left(R^{n}\right)$ modulo $\mathscr{H}\left(C^{n}\right)$.

Proof. We give two proofs. The first is in the spirit of Fourier analysis and is, essentially, a confirmation of Remark 2. The second proof is direct and is, essentially, due to Carleman (see Remark 1).

We claim that the topology of $\hat{\mathscr{E}}^{\prime}$ is defined by $\hat{\mathscr{H}}^{\prime}$ in all of $C^{n}$ and $\hat{\mathscr{E}}{ }^{\prime}$ on $R^{n}$. This is another way of saying that $R^{n}$ is sufficient for $\hat{\mathscr{E}}$ modulo $\hat{\mathscr{H}}$. If we establish our claim then (3.1) follows. For, the claim means that if we map $\left.\hat{\mathscr{E}}^{\prime} \rightarrow \hat{\mathscr{H}}^{\prime} \oplus \hat{\mathscr{E}}^{\prime}\right|_{R}$ by

$$
\hat{S} \rightarrow(\hat{S}, \hat{S})
$$

then $(A, B) \rightarrow \hat{f} \cdot A+\hat{f} \cdot B$ is continuous on the image in the topology of $\left.\hat{\mathscr{H}}^{\prime} \oplus \hat{\mathscr{E}}^{\prime}\right|_{R}$ for any $\hat{f} \in \hat{\mathscr{E}}$. The representation (3.1) then follows from the Hahn-Banach Theorem.

Let us show how to verify the claim. Let $\hat{S} \in \hat{\mathscr{D}}^{\prime}$ be small in the topologies of $\hat{\mathscr{H}}^{\prime}$ and $\left.\hat{\mathscr{E}}^{\prime}\right|_{R}$. Suppose that $\hat{S}$ belongs to bounded sets in these spaces. Thus $\hat{S}$ satisfies

$$
|\hat{S}(\hat{x})| \leqslant\left\{\begin{array}{l}
c^{\prime} \exp c|\hat{x}| \quad \text { in all } C^{n}, \\
c_{0}(1+|\hat{x}|)^{c_{1}} \text { on } R^{n} .
\end{array}\right.
$$

We are writing $|\hat{x}|=\left|\hat{x}_{1}\right|+\cdots+\left|\hat{x}_{n}\right|$.

It is a little simpler to assume $c_{1}=0$. This technicality is easily dispensed with by a regularization argument as in FA.

We claim that (3.4) for $c_{1}=0$ implies

$$
|\hat{S}(\hat{x})| \leqslant c_{0} \exp (c|\operatorname{Im} \hat{x}|)
$$

showing that the set of $\hat{S}$ satisfying (3.4) is bounded in the topology of $\hat{\mathscr{E}}^{\prime}$. This shows that the topology of $\hat{\mathscr{E}}^{\prime}$ is correctly described from the point of view of bounded sets; they suffice for the topology of $\hat{\mathscr{E}}^{\prime}$ because $\mathscr{E}$ is a Fréchet space. (In any case we could use the technique of FA to modify the proof we shall give to apply to neighborhoods instead of bounded sets.) 
Let us fix $\hat{x}_{1}^{0}, \ldots, \hat{x}_{n-1}^{0}$, to be real and let $\hat{x}_{n}$ vary. In this complex plane

$$
\left|\hat{S}\left(\hat{x}_{1}^{0}, \ldots, \hat{x}_{n-1}^{0}, \hat{x}_{n}\right)\right| \leqslant \begin{cases}c^{1} \exp \left(c\left|\hat{x}_{n}\right|\right) & \text { for all } \hat{x}_{n}, \\ c_{0} & \text { for } \hat{x}_{n} \text { real. }\end{cases}
$$

By Phragmèn-Lindelöf we derive

$$
\left|\hat{S}\left(\hat{x}_{1}^{0}, \ldots, \hat{x}_{n-1}^{0}, \hat{x}_{n}\right)\right| \leqslant c_{0} \exp \left(c\left|\operatorname{Im} \hat{x}_{n}\right|\right)
$$

for all $x_{n}$.

Next fix $\hat{x}_{1}^{0}, \ldots, \hat{x}_{n-2}^{0}$ real and $\hat{x}_{n}^{0}$ arbitrary and let $\hat{x}_{n-1}$ vary. Because of (3.7) we have

$$
\left|\hat{S}\left(\hat{x}_{1}^{0}, \ldots, \hat{x}_{n-2}^{0}, \hat{x}_{n-1}, \hat{x}_{n}^{0}\right)\right| \leqslant \begin{cases}c^{2} \exp \left(c\left|\hat{x}_{n-1}\right|\right), & \text { all } \hat{x}_{n-1}, \\ c_{0} \exp \left(c\left|\operatorname{Im} \hat{x}_{n}^{0}\right|\right), & \hat{x}_{n-1} \text { real. }\end{cases}
$$

Since $\hat{x}_{n}^{0}$ is fixed we again apply Phragmèn-Lindelöf to obtain

$$
\left|\hat{S}\left(\hat{x}_{1}^{0}, \ldots, \hat{x}_{n-2}^{0}, \hat{x}_{n-1}, \hat{x}_{n}^{0}\right)\right| \leqslant c_{0} \exp \left(c\left|\operatorname{Im} \hat{x}_{n}^{0}\right|+c\left|\operatorname{Im} \hat{x}_{n-1}\right|\right) .
$$

Proceeding in this way we derive (3.5) hence Theorem 3.1.

For the second proof let us consider first the case $n=1$. We approximate $f$ on $[-2,2]$ by a polynomial $P_{1}$, say $\left|f-P_{1}\right| \leqslant 1 / 2$. Then we want to approximate $f$ on $[-3,3]$ by a polynomial $P_{2}$. But we must control $P_{2}$ in the complex plane to hope to get convergence to an entire function.

We approximate on the complex disc with spurs

$$
\begin{aligned}
& x \text { complex, } \quad|x| \leqslant 1 \text { approximate } P_{1}, \\
& x \text { real, } \quad 1 \leqslant|x| \leqslant 3 \text { approximate } f .
\end{aligned}
$$

Of course, this approximation is not possible because this function is not continuous at $x= \pm 1$. Thus we modify $f$ a little near $x= \pm 1$ to obtain a function $\tilde{f}$ which equals $P_{1}$ at $x= \pm 1$ and differs little from $f$. This is possible since $\left|P_{1}-f\right| \leqslant 1 / 2$ on $-2 \leqslant x \leqslant 2$.

We now approximate $P_{1}, \tilde{f}$ on this region by $P_{2}$ where the approximation is within $2^{-2}$. This approximation is possible because (3.10) is a Runge domain. Note that $P_{2}$ is close to $P_{1}$ on the complex disc $|x| \leqslant 1$.

Proceeding in this way we see that $P_{j}$ converge to an entire function which differs from $f$ by a bounded function. By including derivatives and being more careful in the approximation process we can construct an entire $h$ such that $f-h$ belongs to the space $\mathscr{S}$ of L. Schwartz.

The proof for $n>1$ proceeds along the same lines. We use regions which are unions of a complex ball with a spur defined by

$$
\begin{aligned}
& x \text { complex, } \quad \sum\left|x_{j}\right|^{2} \leqslant l^{2}, \\
& x \text { real, } \quad l^{2} \leqslant \sum\left|x_{j}\right|^{2} \leqslant(l+2)^{2} .
\end{aligned}
$$

Unfortunately, (3.11) is not a domain of holomorphy so we have to compute its envelope of holomorphy. This modifies the ball near the real space, but by only a little. Thus we approximate $P_{l}$ on the complex part of the envelope of holomorphy 
and $f$ (slightly modified) on what remains of the spurs. This approximation can be carried out because the envelope of holomorphy is a Runge domain and because $f$ is close to $P_{l}$ where the spur meets the envelope of holomorphy.

We shall not carry out the details of computing the envelope of holomorphy and checking the Runge property as they are not germain to what follows.

REMARK. It is tempting to replace (3.11) by products of sets of the form (3.10) since these are domains of holomorphy and Runge properties of product sets are obvious. But this method does not seem to work because we do not know how to modify $f$ near the boundary of the spurs.

It is possible to replace the hypothesis $f \in C^{\infty}\left(R^{n}\right)$ by $f \in C^{\infty}(\Omega)$ where $\Omega$ is a closed smooth convex set or a closed polyhedron. But that is because such an $f$ extends to be $C^{\infty}$ on all of $R^{n}$ so the previous result applies. For this reason such a general result cannot apply to solutions of $\mathbf{D} f=0$ which is what we really need.

Let us restrict our considerations to the types of $\Omega$ used in the edge-of-the-wedge theorem. These are of the form $\{(t, y)\}$ where $y \in Y$ is a real linear space and $t \in \Gamma$ which is a closed convex cone. We should like to prove an analog of Theorem 3.1 for solutions of $\mathbf{D} f=0$ on $\Omega$. This is

Problem 3.1. Can every solution of $\mathbf{D} f=0$ on $\Omega$ be written in the form (3.1) where $h$ is an entire solution of $\mathbf{D} h=0$ on all $R^{n}$ and $g$ is an "ordinary" Fourier transform in the sense that (3.2) holds where the support of $\mu$ is contained in the set of $(\hat{t}, \hat{y}) \in V$ with $\hat{y}$ real.

Multiplicities are allowed when $V$, considered as a covering of $\hat{Y}$, has branching over real points. If we make the assumption that the branching over the real $\hat{Y}$ is compact then we can absorb the part of (3.2) over a large disc into $h$ so the branching is generally of no concern to us.

We cannot give a complete solution to this problem. Such a solution would greatly simplify our work in the succeeding chapters. We shall prove the result for the Cauchy-Riemann system and also for elliptic systems when $\operatorname{dim} V=1$. (For $\operatorname{dim} V=$ 0 all solutions are global so there is no problem.)

In order to clarify the situation, let us suppose that $\Omega$ is defined as the set of $(t, y)$ where $t$ lies in the closed positive orthant $\Gamma^{+}$. The description of $\hat{W}^{\prime}=\hat{\mathscr{E}}^{\prime}(\Omega)$ and its topology is given in (1.14) ff. Thus we know what $\hat{W}^{\prime}(V)$ is.

Let us proceed as in the proof of Theorem 3.1 for $\mathbf{D}$ the Cauchy-Riemann system and see where we get into trouble. We suppose we have a set of $\hat{S}$ in $\hat{W}^{\prime}(V)$ which is bounded in both the $\hat{\mathscr{H}}^{\prime}$ norms ( $\mathscr{H}=$ space of entire functions) and the $\hat{W}^{\prime}(V)$ norms on the points of $V$ above real $\hat{Y}$. Using (1.14) this means that, on $V$,

$$
|\hat{S}(\hat{t}, \hat{y})| \leqslant\left\{\begin{array}{l}
c_{0} \exp c|\hat{y}|, \quad \text { all } \hat{t}, \hat{y}, \\
c_{0} \exp c \sum \max \left(0,-\hat{y}_{j}\right), \quad \hat{y} \text { real. }
\end{array}\right.
$$

Note that we ignore the polynomial factor, as before.

We want to prove that this implies

$$
|\hat{S}(\hat{t}, \hat{y})| \leqslant c_{0}^{\prime} \exp c^{\prime}\left[|\operatorname{Im} \hat{y}|+\sum \max \left(0,-\operatorname{Re} \hat{y}_{j}\right)\right]
$$

on $V$ for some $c_{0}^{\prime}, c^{\prime}$ which depend only on $c_{0}, c$. 
Fix $\hat{y}_{1}^{0}, \ldots, \hat{y}_{m-1}^{0}$ real and consider the complex plane where $\hat{y}_{m}$ varies $(m=$ $\operatorname{dim} Y)$. As $\hat{y}_{m}$ varies, the points $\left(\hat{t}, \hat{y}_{1}^{0}, \ldots, \hat{y}_{m-1}^{0}, \hat{y}_{m}\right) \in V$ sweep out a curve $\lambda$ which can be described as the projection of $V$ on $\hat{y}_{1}=\hat{y}_{1}^{0}, \ldots, \hat{y}_{m-1}=\hat{y}_{m-1}^{0}$. More precisely, $\lambda$ is defined by $\hat{t}_{1}=i \hat{y}_{1}^{0}, \ldots, \hat{t}_{m-1}=i \hat{y}_{m-1}^{0}$ fixed and $\hat{t}_{m}=i \hat{y}_{m}$. Call $a^{0}, a^{1}$ the constants

$$
\begin{gathered}
a^{0}=\exp c \sum_{1}^{m-1} \max \left(0,-\hat{y}_{j}^{0}\right), \\
a^{1}=\exp c \sum_{1}^{m-1}\left|y_{j}^{0}\right| .
\end{gathered}
$$

Then if we use $\hat{y}_{m}$ as a parameter on $\lambda$ the bounds (3.12) are of the form

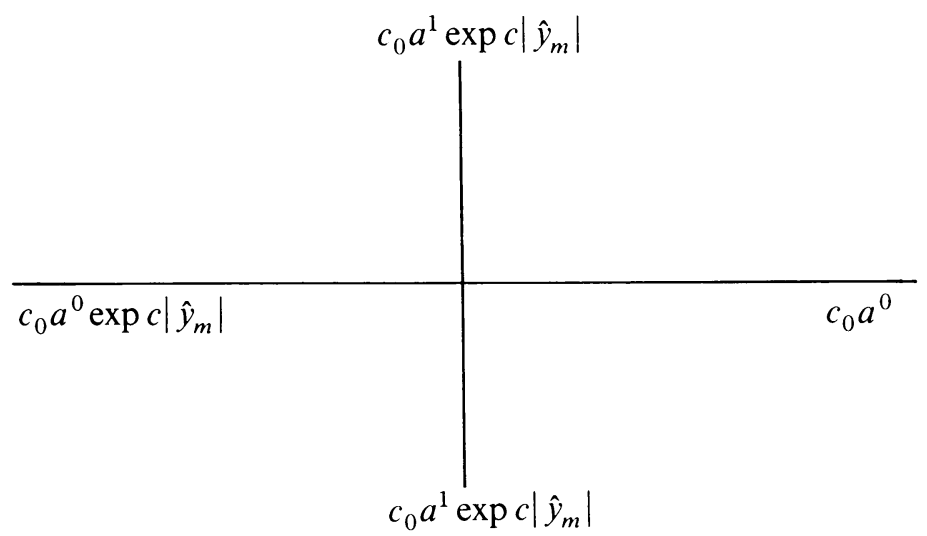

We want to obtain the bounds given by (3.13) which are

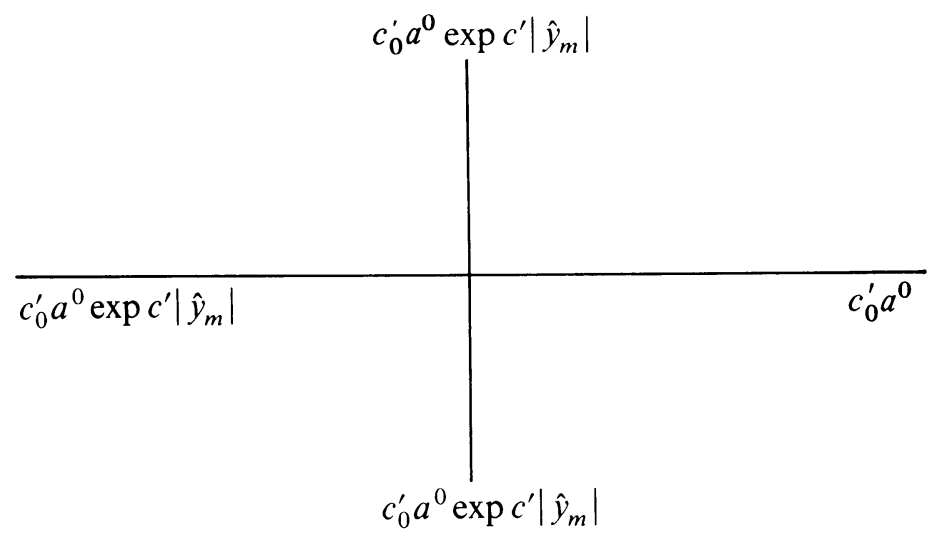

Thus we want to get rid of the annoying factor $a^{1}$. It is tempting to apply the Phragmèn-Lindelöf method as we did in the proof of Theorem 3.1. However in that case we had boundedness on the negative real axis and this time we have exponential growth. 
The failure of the Phragmèn-Lindelöf method to handle this case is made definitive by

Proposition 3.1. ${ }^{1}$ There are no a priori constants $l, m$ so that entire functions $f(x)$ of exponential type 2 which are bounded by 1 on the positive real axis and by $\exp (-x)$ on the negative axis are bounded by $l \exp (m|x|)$ on the imaginary axis.

Proof. We set

$$
g(x)=f(x) e^{x / 2}
$$

Thus $g(x)$ is bounded by $\exp |x| / 2$ on the whole real axis.

We now consider the space $U$ of entire functions which are bounded by $\exp \operatorname{Re}|x| / 2$. The Fourier transform of the dual consists of functions which are regular in the strip $|\operatorname{Im} \hat{x}|<1 / 2$. This means that the linear combinations of $\delta_{0}^{(m)}$ are dense in $\hat{U}$. Hence the polynomials are dense in $U$.

The functions $\exp \left(i m^{\prime} x\right)$ belong to $U$ for any $m^{\prime}$. By the above, we can approximate them in the topology of $U$ by polynomials $P_{j}$. Hence $P_{j}$ cannot be bounded by $l \exp (m|\operatorname{Im} x|)$ for any $l, m$. Since

$$
\left|P_{j}(x)-e^{i m^{\prime} x}\right| / e^{|x| / 2} \rightarrow 0
$$

uniformly on the real $x$ axis it follows that

$$
\left|P_{j}(x)\right| e^{-|x| / 2} \leqslant 2
$$

for $j$ large enough. This means that the functions $P_{j}(x) e^{-x / 2} / 2$ satisfy the hypothesis of Proposition 3.2 but are not bounded by $l \exp (m|x|)$ for any $l, m$. Proposition 3.2 is thereby proven.

Instead of using an induction based on setting $\hat{y}_{j}=$ constant, we could try to prove the result by examing all complex lines through the origin in $\hat{y}$ space. This is akin to making a type of Radon transform (see [5]). While this method has some merit, there are difficulties which we cannot overcome; these are of the same nature as the Radon transform's not being a topological isomorphism on the space $\mathscr{D}$ (see [5] for details of this phenomenon). However this method is promising because we can use all complex lines rather than only real lines which are relevent for Radon transform.

Another hope is the development of a truly multidimensional Phragmèn-Lindelöf theory.

Although the method of our first proof fails in general, we can still apply the second method of proof to the Cauchy-Riemann system. Moreover the first proof works in case $\operatorname{dim} V=1$.

THEOREM 3.3. Suppose $V$ is the Cauchy-Riemann variety for general $n$ or that $\operatorname{dim} V=1$. Then Problem 3.1 has a positive solution.

Proof. Let us start with the case of $\operatorname{dim} V=1$. We may assume, for simplicity of notation, that all the branching of $V$, considered as a covering of $\hat{Y}$, occurs in $|\hat{y}|<1$. We shall show that the real $\hat{Y}$ axis union with $|\hat{y}|=1$ is sufficient for $\hat{\mathscr{E}}^{\prime}$

\footnotetext{
${ }^{1}$ This was observed independently by B. A. Taylor.
} 
modulo $\hat{\mathscr{H}}^{\prime}$. Since the Fourier transform of a compactly supported measure is entire this suffices for us.

We consider each $\hat{t}_{j}$ as a multivalued function of $\hat{y}$ in $|\hat{y}| \geqslant 1$. Since $\hat{Y}$ is noncharacteristic it follows from Chapter IX of FA that

$$
\left|t_{j}(\hat{y})\right| \leqslant c(1+|\hat{y}|)
$$

for all $j$ and, moreover the $\hat{t}_{j}$ are integral over the ring of polynomials in $\hat{y}$. This means that for each $j$ the Puiseaux expansions of the various branches of $\hat{t}_{j}$ at $\hat{y}=\infty$ take the form

$$
\hat{t}_{j}(\hat{y})=\sum_{i=-\infty}^{l} a_{j}^{i} \hat{y}^{i / l}
$$

for some positive integer $l$. By chosing $l$ large enough we can make it independent of $j$ and of the branch. But the $a_{j}^{i}$ might still depend on the branch.

We interpret $\hat{y}^{1 / l}$ as follows: Start with the principal branch on the positive $\hat{y}$ axis. As the positive $\hat{y}$ axis turns counterclockwise to the negative $\hat{y}$ axis the set $0 \leqslant \hat{y}<\infty$ sweeps out regions on $V$ by use of (3.20). These regions cover $|\hat{y}| \geqslant 1$, Im $\hat{y} \geqslant 0$. Then as we continue to sweep to come back to the positive real axis we obtain regions covering $|\hat{y}| \geqslant 1, \operatorname{Im} \hat{y} \leqslant 0$. Continuing in this way we obtain a disection of $V$ into regions $A^{k}$ which are defined as those regions which lie over the parts of the quadrants in the complex $\hat{y}$ plane lying in $|\hat{y}| \geqslant 1$. For each of these regions the function $\hat{y}^{i / l}$ is well defined.

Now pick a fixed region $A^{k}$. Let us examine what happens as $\hat{y} \rightarrow+\infty$ (or $-\infty$ as the case may be) through real values. There are three possibilities:

(a) $a_{j}^{l}$ is not real and $\operatorname{Im} a_{j}^{l}>0$.

(b) $a_{j}^{l}$ is not real and $\operatorname{Im} a_{j}^{l}<0$.

(c) $a_{j}^{l}$ is real (including the possibility that $a_{j}^{l}=0$ ).

Suppose first we are in the case $\hat{y} \rightarrow+\infty$ and (b) holds for some $j$. Then on the positive real $\hat{y}$ axis and, in an angle around it, $\exp \left(-\operatorname{Im} t_{j}(\hat{y})\right)$ grows exponentialiy. This exponential growth is not cancelled by anything else so the norms in $A^{k}$ defined by $\hat{\mathscr{E}}^{\prime}$ are the same as those of $\hat{\mathscr{H}}^{\prime}$.

The same is true if $\hat{y} \rightarrow-\infty$ and (a) holds for some $j$.

Next consider the case $\hat{y} \rightarrow+\infty$ and (a) or (c) holds for all $j$ [which is the same as $\hat{y} \rightarrow-\infty$ and (b) or (c)]. For those $j$ for which (a) holds $\operatorname{Im} \hat{t}_{j}(\hat{y})$ is positive near the positive real $\hat{y}$ axis so the $\hat{t}_{j}$ part of the norm is not important.

Finally suppose we are in case (c). Then on the real $\hat{y}$ axis it is clear from (3.20) that $\operatorname{Im} \hat{t}_{j}$ will grow like some power of $|\hat{y}|$, say

$$
\operatorname{Im} \hat{t}_{j} \sim a_{j}|\hat{y}|^{\alpha_{j}}
$$

as $|\hat{y}| \rightarrow \infty$ in $A^{k}, \hat{y}$ real. Only the case $a_{j}<0, \alpha_{j}>0$ is of any interest. In that case the $\hat{t}_{j}$ coordinate contributes $\exp \left(c|\hat{y}|^{\alpha_{j}}\right)$ to the norm over the real axis.

Now $0<\alpha_{j}<1$ so we can apply the Phragmèn-Lindelöf principle to $A^{k}$ to obtain bounds

$$
c_{0}(1+|\hat{y}|)^{c_{1}} \exp \left(c_{2}|\hat{y}|^{\alpha}+c_{3}|\operatorname{Im} \hat{y}|\right)
$$


on $A^{k}$ for functions which belong to a set $B$ in $\hat{\mathscr{E}}^{\prime}(V)$ which is bounded in the topology of $\hat{\mathscr{H}}^{\prime}$ on all of $V$ and in the topology of $\hat{\mathscr{E}}^{\prime}(V)$ over the real part of $\hat{Y}$. Here $\alpha$ is the maximum of the $\alpha_{j}$ of (3.21) and $c_{0}, c_{1}, c_{2}$ depend on the bounds of $B$ given by $\hat{\mathscr{E}}^{\prime}$ over real $\hat{y}$ and $c_{3}$ depends on the bounds of $B$ given by $\hat{\mathscr{H}}^{\prime}$.

To complete the proof of Theorem 3.3 for $\operatorname{dim} V=1$ we need the following observation:

$$
|\hat{y}|^{\alpha}+|\operatorname{Im} \hat{y}| \leqslant \beta\left(|\operatorname{Im} \hat{y}|+\sum \max \left(1,\left|\operatorname{Im} \hat{t}_{j}\right|(\hat{y})\right)\right.
$$

in $A^{k}$ for some constant $\beta$. Our construction shows that (3.23) holds for real $\hat{y}$. Since $\beta \operatorname{Im} \hat{y}$ varies faster than $\operatorname{Im} \hat{y}^{\alpha}$ for any $\alpha<1$ and faster than $\operatorname{Im} \hat{t}_{j}$ the same result holds for all large $\hat{y}$ in $A^{k}$. Thus $B$ is bounded in $\hat{\mathscr{E}}^{\prime}(V)$.

This completes the proof of Theorem 3.3 regarding varieties of dimension 1 .

Remark. For elliptic systems we could avoid case (c), which is the most difficult case, by making a generic change of variables in $t$. This might diminish $\Gamma^{+}$ somewhat but that is of little consequence.

Next let us prove the result for the Cauchy-Riemann variety. As we have remarked above it seems difficult to give a Fourier analysis proof because of Proposition 3.2. Thus a Fourier analysis proof would necessitate a multidimensional Phragmèn-Lindelöf theorem which is not (in any obvious way) reducible to the one dimensional theorem. This is a technique that is beyond me at present.

Since the details are cumbersome and, presumably, "known" to experts, I shall explain how to modify the second proof of Theorem 3.1 to fit the present situation.

Consider first the case $n=1$. We now approximate $f$ on the intersection of $|x| \leqslant 2$ with the upper half-plane by a polynomial, say $\left|f-P_{1}\right| \leqslant 1 / 2$. We approximate $P_{1}$ on the complex disc with spurs

$$
\begin{gathered}
x \text { complex, }|x| \leqslant 1 \text { approximate } P_{1}, \\
\operatorname{Im} x \geqslant 0, \quad 1 \leqslant|x| \leqslant 3 \text { approximate } f .
\end{gathered}
$$

Unlike the analogous situation arising out of (3.10) we cannot modify $f$ to make it equal to $P_{1}$ on the overlap set. We thus have to apply Runge approximation directly to the function $g_{1}$ which is $f$ on $\operatorname{Im} x \geqslant 0,1 \leqslant|x| \leqslant 3$ and $P_{1}$ on $|x| \leqslant 1, \operatorname{Im} x \leqslant 0$. To approximate $g_{1}$ we replace $g_{1}$ by its Cauchy integral over the boundary of this region. See Figure 1.

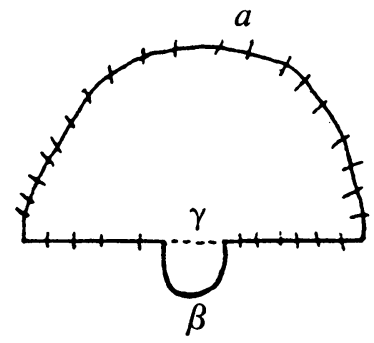

FIGURE 1 
Since $g_{1}=f$ on $\alpha$ we can use Cauchy's theorem to replace $\alpha$ by $\gamma$ for points in the region bounded by $\gamma$ and $\beta$ where we use $f$ on $\gamma$ and $P_{1}$ on $\beta$ in the Cauchy integral. Since $f \sim P_{1}$ on $\gamma$ this Cauchy integral is close to $P_{1}$ in this region. Similarly the Cauchy integral is close to $f$ in the region bounded by $\alpha$ and $\gamma$.

We now approximate the Cauchy integral of $g_{1}$ by a polynomial $P_{2}$. Proceeding in this way we obtain global approximation in case $n=1$.

For $n>1$ we have to make a similar modification of (3.11). We must then estimate the size of the resulting envelope of holomorphy and use the Cauchy-Weil integral to replace our above construction in $n=1$.

As we mentioned above, since the details are complicated and we make very little use of the result we shall not give any more details.

4. Operational calculus and balayage. To understand what operational calculus is all about, let us prove the simplest (noncompact) form of the edge-of-the-wedge theorem, namely for functions of one complex variable, by means of Fourier analysis.

We are thus given two functions $f^{ \pm}(t, y)$ which are holomorphic in $t>0$ (respectively $t<0$ ) and agree on the real axis $t=0$. To be pedantic, denote by $f_{0}^{ \pm}(y)$ the Cauchy data of $f^{ \pm}$. Thus $f_{0}^{+}=f_{0}^{-}$but we shall ignore this equality for the present.

By Theorem 3.1 we may assume that $f_{0}^{ \pm}$are Fourier transforms in the usual sense. Of course we must pay for this by replacing the equality $f_{0}^{+}=f_{0}^{-}$by $f_{0}^{+} \equiv f_{0}^{-}$ where the congruence refers to congruence modulo entire functions.

In order to make things precise we use the notation

$$
f(y)=\int \hat{f}(\hat{y}) e^{i y \hat{y}} d \hat{y}, \quad \hat{f}(\hat{y})=\int f(y) e^{-i y \hat{y}} d y .
$$

Although $f_{0}^{+}$is the $C D$ of a function which is analytic in the upper half-plane we cannot see this from its Fourier transform $\hat{f}_{0}^{+}$. This means that $\hat{f}_{0}^{+}$may not be exponentially decreasing as $\hat{y} \rightarrow-\infty$. This is because $f_{0}^{+}$may not be small on $t=$ constant, except for $t=0$.

In the compact case we used the pointwise identity $\hat{f}^{+}(\hat{y})=\hat{f}^{-}(\hat{y})$ to deduce the smallness of each of $\hat{f}^{ \pm}(\hat{y})$. This was possible because for each direction at infinity one of $\hat{f}^{ \pm}(\hat{y})$ was small. As we have just mentioned, the analyticity properties of $f^{ \pm}$ do not follow from the smallness properties of $\hat{f}^{ \pm}$but from more subtle "cancellation" property. Our proof of the edge-of-the-wedge theorem works because the cancellation properties as $\hat{y} \rightarrow \pm \infty$ are independent; we can make use of this independence to replace the smallness property.

The above remarks suggest considering $\hat{\chi}^{ \pm} \hat{f}^{+}$and $\hat{\chi}^{ \pm} \hat{f}^{-}$where $\hat{\chi}^{ \pm}$is $i$ times the characteristic function of $\hat{y} \geqslant 0$ (resp. $\hat{y} \leqslant 0$ ). The Fourier transform of $\hat{\chi}^{+} \hat{f}_{0}^{+}$is certainly holomorphic in $t>0$. Since $i \hat{f}_{0}^{+}=\left(\hat{\chi}^{+}+\hat{\chi}^{-}\right) \hat{f}_{0}^{+}$the same is true of $\hat{\chi}^{-} \hat{f}_{0}^{+}$. Similarly the Fourier transforms of $\hat{\chi}^{ \pm} \hat{f}_{0}^{-}$are holomorphic in $t<0$.

We now use our hypothesis that $f_{0}^{+} \equiv f_{0}^{-}$. This means that $\hat{f}_{0}^{+}-\hat{f}_{0}^{-}$is the Fourier transform of an entire function. As above this means that the Fourier transform of $\hat{\chi}^{+}\left(\hat{f}_{0}^{+}-\hat{f}_{0}^{-}\right)$is holomorphic in both $t>0$ and $t<0$. In particular, the Fourier transform of $\hat{\chi}^{+} \hat{f}_{0}^{+}$is holomorphic in $t<0$. 


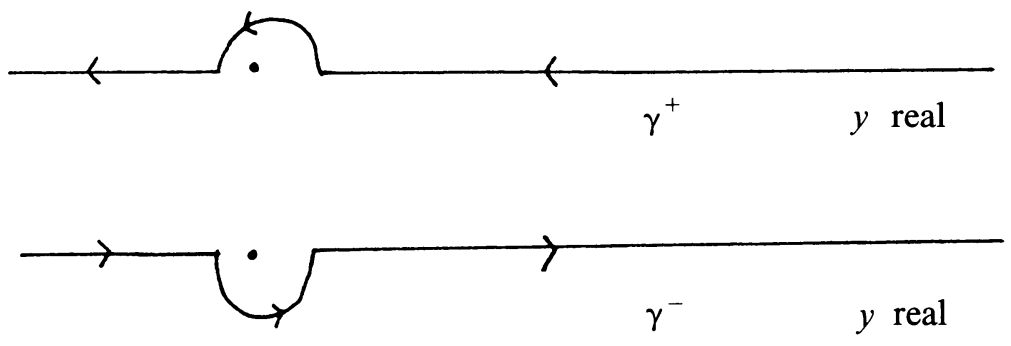

FIGURE 2

We do not seem to have gained anything because the Fourier transform of $\hat{\chi}^{+} \hat{f}_{0}^{+}$ is holomorphic in $t>0$ and $t<0$. If we knew that this implies that the Fourier transform of $\hat{\chi}^{+} \hat{f}_{0}^{+}$is entire then we could also say that the Fourier transform of $\hat{\chi}^{-} \hat{f}_{0}^{+}$is entire which implies that $f_{0}$ is entire.

But it seems that we need the edge-of-the-wedge theorem to conclude that the Fourier transform of $\hat{\chi}^{+} \hat{f}_{0}^{+}$is entire!

However, this is not the case. We can use the special nature of $\hat{\chi}^{+}$to guarantee that $\hat{\chi}^{+} \hat{f}_{0}^{+}$has an entire Fourier transform.

Let us observe that $\chi^{+}=1 / y$ so $\chi^{+} * f_{0}^{+}$is exactly the Cauchy integral of $f_{0}^{+}$. We must be careful to get the exact definition of $\chi^{+}$as this is crucial for what follows and also accounts for the distinction between $\chi^{+}$and $\chi^{-}$. We thus use the contour $\gamma^{ \pm}$for $\chi^{ \pm}$where we have the situation in Figure 2.

Proposition 4.1. If $f$ is holomorphic in $t<0$ and small at infinity on $t=0$ then $\chi^{+} * f$ is entire. $\left(\chi^{+} * f\right.$ refers to convolution on $t=0$.)

By "small at infinity" it suffices that $f(y)=O\left(|y|^{-2}\right)$.

For the application we set $\hat{f}=\hat{\chi}^{+} \hat{f}^{+}$and we also use the analog of Proposition 4.1 with $t<0$ replaced by $t>0$ and $\hat{f}=\hat{\chi}^{-} \hat{f}^{-}$.

Proof. We write

$$
\left(\chi^{+} * f\right)(y)=\int_{\gamma^{+}} f\left(y-y^{\prime}\right) \frac{d y^{\prime}}{y^{\prime}}=\int_{\gamma_{y}^{-}} \frac{f\left(y^{\prime}\right) d y^{\prime}}{y-y^{\prime}}
$$

where $\gamma_{y}^{-}$means $\gamma^{-}$translated by $y$.

In (4.1) we start with real $y$. We replace $\gamma_{y}^{-}$by $\lambda\left(\gamma^{-}\right)$which equals $\gamma^{-}$at infinity but has an increased circular part. This can be done since $f$ is holomorphic in the lower half-plane and small at infinity. It is clear that the integral is holomorphic as long as $y$ is above $\lambda\left(\gamma^{-}\right)$. It follows that $\chi^{+} * f$ is entire, which is the desired result.

We can interpret Proposition 4.1 as stating that $\hat{y}>0$ can be balayaged out of the support of $\hat{f}$ without changing the congruence class of $f$ modulo entire functions. We can also state the result in terms of support in the sense of hyperfunction theory.

Let us examine how the above proof of Proposition 4.1 works for functions of several complex variables. Suppose $f$ is holomorphic for $t_{1}<0, \ldots, t_{r}<0$ and small at infinity on $t=0$. Let $\hat{\chi}^{+}$be the characteristic function of the positive orthant in real $\hat{y}$ space. To show that $\chi^{+} * f$ is entire we should try to integrate in the complex 
$y_{1}$ plane. But once we go off the real $y_{1}$ axis we need to know that $f_{0}\left(y_{1}, y_{2}, \ldots, y_{r}\right)$ is small at infinity for this $y_{1}$ and $y_{2}, \ldots, y_{r}$ real and large. Unfortunately Theorem 3.1 does not give such a strong result so we cannot prove the analog of Proposition 4.1 for functions of several complex variables by the method thus far presented.

The failure of this proof is another indication of the major distinction that exists between holomorphic functions of one and more than one complex variable.

Problem 4.1. Develop a theory of change of contour in several complex variables that would allow to change to contour from real $Y$ to replace an integral over the boundary of large compact sets in $Y$ by integrals in suitable regions of the complexification of $Y$.

A suitable solution to this problem would enable us to convolve directly with $\chi^{ \pm}$ where $\hat{\chi}^{ \pm}$is the characteristic function of the positive (negative) orthant and to prove the analog of Proposition 4.1 in higher dimension.

Because of our inability to deal successfully with Problem 4.1, most of our considerations are restricted to varieties of dimension 1.

Let us go back to Proposition 4.1. What property of $\chi^{+}$was needed to obtain the conclusion?

Proposition 4.2. Let $\xi^{ \pm}$be any function of real $y$ which extends to a function of complex $y$ which is holomorphic and bounded in the exterior of a closed cone $\Gamma^{\mp}$ contained in the interior of the lower (upper) half-plane $\cup\{0\}$. If $f^{ \pm}$is holomorphic in $\operatorname{Im} y<0($ resp. $\operatorname{Im} y>0)$ and small at infinity on the real axis than $\xi^{ \pm} * f^{ \pm}$is entire.

Proof. We give the proof for $\xi^{+}$as the proof for $\xi^{-}$is similar. As in (4.1) ff we write

$$
\begin{aligned}
\left(\xi^{+} * f\right)(y) & =\int_{\gamma^{+}} f\left(y-y^{\prime}\right) \xi^{+}\left(y^{\prime}\right) d y^{\prime} \\
& =\int_{\lambda\left(\gamma^{-}\right)} f\left(y^{\prime}\right) \xi^{+}\left(y-y^{\prime}\right) d y^{\prime}
\end{aligned}
$$

for $y$ real. The integral over $\lambda\left(\gamma^{-}\right)$involves values of $\xi^{+}$on the contour $y-\lambda\left(\gamma^{-}\right)$. See Figure 3.

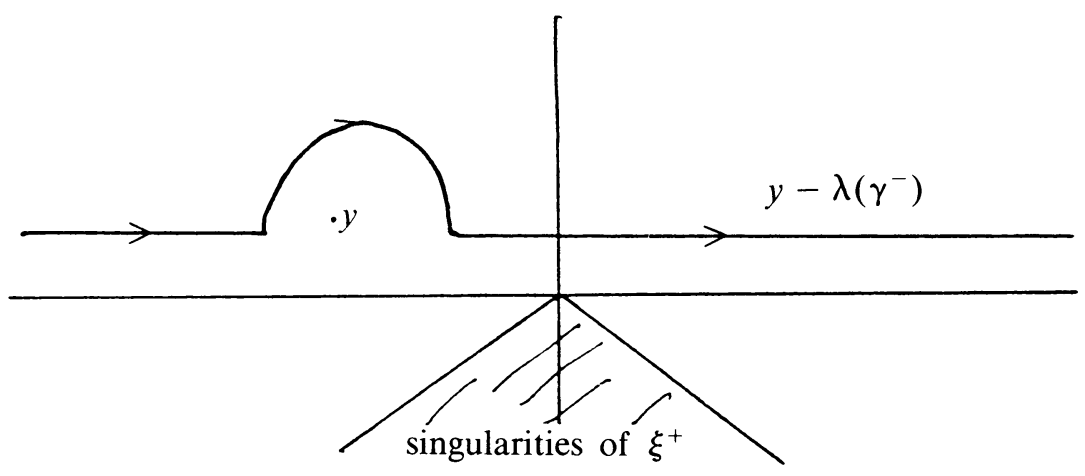

FIGURE 3 
It is clear that for any complex $y$ we can make the semicircle in $\lambda\left(\gamma^{-}\right)$so large that the entirety of $y-\lambda\left(\gamma^{-}\right)$lies above the singularities of $\xi^{+}$. The integral is clearly an entire function of $y$ which is the desired result.

REMARK. To make a similar argument in higher dimensions we might need a function $\xi^{+}$which is holomorphic and small for all complex $y$ except for a convex cone. Such a $\xi^{+}$would necessarily be entire by standard extension theory. Hence $\xi^{+}$ would be a polynomial which is of no interest to us.

However $\bar{\partial}$ closed one forms with the desired properties do exist and they should ameliorate the need for holomorphic functions $\xi^{ \pm}$.

The usefulness of Proposition 4.2 comes from

Proposition 4.3. Let $\hat{\xi}$ be an algebraic function of $\hat{y}$ whose branch points lie in $|\hat{y}|<a$. Then

$$
\xi^{ \pm}(y)=\int_{ \pm a}^{ \pm \infty} e^{i y \hat{y}} \hat{\xi}(\hat{y}) d \hat{y}
$$

satisfies the hypothesis of Proposition 4.2.

Proof. We consider $\xi^{+}$as $\xi^{-}$is treated similarly. The integral certainly defines $\xi^{+}$ as a holomorphic function in the upper half-plane. Moreover, $\xi^{+}$is bounded in any closed cone contained in the open upper half-plane.

For suitable $y$ in the upper half-plane we can shift the contour in (4.3) to $\arg (\hat{y}-a)=\theta$ for sufficiently small $\theta$. But such an integral is holomorphic and small in $|\arg y-\theta|<\pi / 2$. This is Proposition 4.3.

Remark 1. The proof shows that the cone $\Gamma^{\mp}$ of Proposition 4.2 can be an arbitrarily "thin" cone containing the negative (positive) imaginary axis.

REMARK 2. It may be possible to prove some results when $\operatorname{dim} y>1$. The correct hypothesis is that the singular set of $\xi$ should contain no real point at infinity. But we do not know how to make use of such results.

ANOTHER APPROACH. We wish to give another approach to Propositions 4.1, 4.2, and 4.3 as this seems interesting and useful. This approach works with $\hat{f}$ rather than $f$.

Let us start with the Cauchy-Riemann equation. Since $f$ is holomorphic in $t<0$ we can use the Fundamental Principle of FA (Theorem 4.1) to produce a measure $\mu$ on $V$ (the Cauchy-Riemann variety) whose Fourier transform is $f$. The support of $\mu$ is on the complex $V$ (that is, complex $\hat{y}$ when $\hat{y}$ is used as a parameter for $V$ ) thought of as a real two dimensional space; but $\mu$ is exponentially decreasing at infinity outside an angle about the negative real $\hat{y}$ axis. All this follows from our explicit description of $\mathscr{E}(t \leqslant 0)$ given in Chapter 1 .

Of course, we cannot assert that $\mu=\hat{f}$ since, as we have observed many times, there is no uniqueness for complex Fourier representation. However $\hat{f}-\mu$ is orthogonal to all exponentials.

Proposition 4.4. We have

$$
\hat{f}-\mu=\bar{\partial} \nu
$$


where $\nu$ is a distribution having the decrease properties of $\hat{\mathscr{E}}$, that is,

$$
\nu(\hat{y}, \hat{t})=O\left[(1+|\hat{y}|+|\hat{t}|)^{-c} \exp (-c|\hat{t}|)\right]
$$

for all $c$. Here $\hat{y}$ and $\hat{t}$ are real.

REMARK. By a slight sharpening of our methods we could assert that $\mu, \hat{f}$, and $\nu$ are smooth functions, but this is not important for what follows.

Proof. Proposition 4.4 is easily proven using Fourier analysis on the complex $\hat{y}$ plane thought of as a real two dimensional plane.

We can now complete the balayage. For, let $\chi$ be the characteristic function of some angle around the positive $\hat{y}$ axis, or, more precisely let $\chi$ be a smooth cut-off function approximating this characteristic function.

We multiply (4.4) by $\chi$ to obtain

$$
\hat{\chi}^{+} \hat{f}=\chi \hat{f}+\varepsilon=\chi \mu+\chi \bar{\partial} \nu+\varepsilon=\chi \mu+\bar{\partial}(\chi \nu)-(\bar{\partial} \chi) \nu+\varepsilon .
$$

Here $\varepsilon$ has compact support so its Fourier transform is entire. Moreover, $\mu$ is exponential decreasing in an angle around the positive $\hat{y}$ axis, hence on the support of $\chi$ so $\chi \mu$ has an entire Fourier transform. The support of $\bar{\partial} \chi$ omits an angle around the $\hat{y}$ axis so $(\vec{\partial} \chi) \nu$ is exponentially decreasing hence has an entire Fourier transform. Finally the Fourier transform of $\bar{\partial}(\chi \nu)$ is zero when we use the complex structure of $V$, since we are using exponentials which are holomorphic in $\hat{y}$. (Of course this Fourier transform is defined only in $t \leqslant 0$.)

This completes the second proof of Proposition 4.1.

A similar method can be used to prove Proposition 4.2. This approach can be used working directly with $\hat{f}$ to prove the following result, which is the main result of this chapter, and which also follows directly from Propositions 4.2 and 4.3.

THEOREM 4.5. Suppose $f(y)$ is an entire function which is a classical Fourier transform of $\hat{f}(\hat{y})$. Let $\hat{\xi}^{ \pm}$be branches of an algebraic function which are defined for $\hat{y}$ real, $\hat{y} \geqslant a($ resp. $\hat{y} \leqslant-a)$. Then the Fourier transform of $\hat{\xi}^{ \pm} \hat{f}$ is entire.

5. The main results. In case $\operatorname{dim} V=1$ the approximation theory of Chapter 3 and the operational calculus of Chapter 4 put us in a position that is as favorable as the compact theory of Chapter 2.

Suppose that $f^{ \pm}$are solutions of $\mathbf{D} f^{ \pm}=0$ in the region where $t \in \Gamma^{ \pm}$and $y$ is arbitrary. Suppose that there is a well-defined Cauchy problem for $\mathbf{D}$ using harmonic polynomials $h_{s}(t)$ as in Chapter 2. We consider the edge-of-the-wedge conditions

$$
h_{s}(\partial / \partial t) f^{+}=h_{s}(\partial / \partial t) f^{-} \text {on } Y .
$$

By the results of Chapter 3 we can assume that $f^{ \pm}$are Fourier transforms on the part of $V$ above real $\hat{Y}$ provided that we replace equality in (5.1) by congruence mod entire functions. Thus (5.1) can be written in the form

$$
\sum_{\hat{t}} \hat{f}^{+}(\hat{t}, \hat{y}) h_{s}(\hat{t}) \equiv \sum_{\hat{t}} \hat{f}^{-}(\hat{t}, \hat{y}) h_{s}(\hat{t}) .
$$


Equation (5.2) is the exact analog of (2.34). We cannot use the method of Chapter 2 to conclude directly that $\hat{f}^{ \pm}$are exponentially decreasing. Rather, we rewrite (5.2) as

$$
\sum\left[\hat{f}^{+}(\hat{t}, \hat{y})-\hat{f}^{-}(\hat{t}, \hat{y})\right] h_{s}(\hat{t})=\hat{g}_{s}(\hat{y}) .
$$

Here $\hat{g}_{s}(\hat{y})$ is small (polynomially) at infinity and $g_{s}(y)$ is entire.

REMARK. If we assume that the functions $f^{ \pm}$are polynomially increasing in $y$ for each $t$ or, more generally, that they belong to the space $S^{\prime}$ of tempered distributions uniformly for $t$ in compact sets, then we can replace congruence in (5.2) by equality and obtain the same results as in the compact case even if $\operatorname{dim} Y>1$. The main difficulties appear because we do not impose any bounds on the solutions $f^{ \pm}$.

Since the $h_{s}(\hat{t})$ span the functions on the points of $V$ above $\hat{y}$ (generically) we can solve (5.3) generically to obtain

$$
\left[\hat{f}^{+}(\hat{t}, \hat{y})-\hat{f}^{-}(\hat{t}, \hat{y})\right]=\sum \hat{H}_{s}(\hat{t}) \hat{g}_{s}(\hat{y}) .
$$

The $\hat{H}_{s}(\hat{t})$ are algebraic functions of $\hat{y}$ since the $h_{s}$ are polynomials in $\hat{t}$ hence are algebraic in $\hat{y}$.

When we are outside of a compact set of $\hat{y}$ say $|\hat{y}| \geqslant a$ the algebraic branches are well defined. We can thus apply Theorem 4.5 to conclude that $\hat{f}^{+}-\hat{f}^{-}$when restricted to a fixed branch of $V$, say when multiplied by $\chi$, over $\hat{y} \geqslant a$ or $\hat{y} \leqslant-a$ has an entire Fourier transform in $\hat{y}$.

In the proof of Theorem 3.3 we discussed the asymptotic behavior of each $\left|\exp \left(i \hat{t}_{j}(\hat{y})\right)\right|$ on the branch in question. It is clear that either $\operatorname{Im} \hat{t}(\hat{y})$ lies asymptotically outside $\hat{\Gamma}^{+} \cup \hat{\Gamma}^{-}$or else it lies in one of $\hat{\Gamma}^{+}$or $\hat{\Gamma}^{-}$. In the first case the growth conditions imposed by both spaces $\hat{\mathscr{E}}^{ \pm}$on the restrictions of $\hat{f}^{ \pm}$to this part of the branch are equal so the Fourier transforms of both of these restrictions are defined and solutions of $\mathbf{D}$ in all $(t, y)$.

In the second case at least one of them, say $\hat{f}^{-}$has a Fourier transform which is defined and a solution of $\mathbf{D}$ in all $(t, y)$.

We now write (5.4) in the form

$$
\chi \hat{f}^{+}(\hat{t}, \hat{y})=\chi \hat{f}^{-}(\hat{t}, \hat{y})+\chi \sum \hat{H}_{s}(\hat{t}) \hat{g}_{s}(\hat{y}) .
$$

The first term on the right has Cauchy data which are Cauchy data of a global solution. The Cauchy data of the second term, which are obtained by multiplying by $\chi \sum \hat{H}_{s}$ are entire by Theorem 4.5. Hence, by the CK (Cauchy-Kowalewski) theory of Chapter IX of FA, the Cauchy data of this term also correspond to a global solution. Thus, the Cauchy data of $\chi \hat{f}^{+}$are Cauchy data of a global solution. By the uniqueness of the Cauchy problem, the Fourier transform of $\chi \hat{f}^{+}(\hat{t}, \hat{y})$ extends to a global solution.

Since this is true for all branches of $V$ we have thus proven the general edge-of-the-wedge result.

Theorem 5.1. Let $\mathbf{D}$ be a system with $\operatorname{dim} V=1$. Suppose $\mathbf{D} f^{ \pm}=0$ in the tube $t \in \Gamma^{ \pm}$. If the Cauchy data for $f^{ \pm}$coincide then there is a unique $f$ defined for all $(t, y)$ and satisfying $\mathbf{D} f=0$ which extends $f^{ \pm}$. 
The proof of Theorem 5.1 shows how to extend the results of Chapter 2 to the present situation. In particular we have (in all cases $\operatorname{dim} V=1$ )

THEOREM 5.2. When $\mathbf{D}^{ \pm}$are elliptic and when the algebraic conditions demanded by Problem 2.1 are satisfied (in $(\hat{t}, \hat{y})$ in place of $(k, l)$ ) then solutions $f \pm$ of $\mathbf{D}^{ \pm} f \pm=0$ on the tube $t \in \Gamma^{ \pm}$with equal Cauchy data extend to solutions in the whole space.

THeORem 5.3. Any set of $q^{+}$of the $h_{s}$ satisfying the requirements of Problem 2.2 gives a reflection theorem in the sense that if the corresponding Cauchy data of $f^{+}$ vanish on $Y$ then $f^{+}$extends to a solution of $\mathbf{D}^{+}$in the whole space.

Theorem 5.4. Suppose $\mathbf{D} f^{j}=0$ in the tube $t \in \Gamma^{j}$ where $\Gamma^{j}$ is a proper convex cone. If the Cauchy data of $f^{j}$ agree on $Y$ then both $f^{j}$ extend to a single solution $f$ on the tube over the convex hull of $\Gamma^{1} \cup \Gamma^{2}$.

Finally, as in Chapter 2 we can use a slight modification of Theorem 5.4 (in which we use only a cut off part of $\Gamma^{j}$ ) to prove the following chain extension result.

THEOREM 5.5. In case $\operatorname{dim} V=1$ then any solution of $\mathbf{D} f=0$ defined near the one skeleton of a cube in T times all of $Y$ extends to a solution over the interior of the cube times all of $Y$.

The argument at the beginning of this chapter leads to the following result which is of independent interest.

THEOREM 5.6. Suppose $\operatorname{dim} V=1$. If $\hat{f}$ has support on the part of $V$ lying over real $\hat{y}$ and $f$ is a global solution of $\mathbf{D} f=0$ then the Fourier transform of the restriction of $\hat{f}$ to any branch of $V$ lying over $\hat{y} \geqslant a$ or $\hat{y} \leqslant-a$ is a global solution.

6. Dual theory. Up to now our work has been concentrated on the space of solutions of $\mathbf{D} f=0$ rather than on the dual space. The reason for this is that the ideas of Chapters 3 and 4 which deal with solutions in tubes modulo entire solutions do not seem to fit naturally into the dual theory. In this chapter we shall explain some ideas that are more natural in the dual theory. We are not able to use these ideas to rederive the results of Chapter 5, but we shall derive some new types of density results as well as results concerning Cauchy data which are taken on in a limit sense as in Part I. We shall also describe new functorial operations of AU intersection and $\mathrm{AU}$ union.

Let us begin by defining the union of two topological vector spaces with amalgamation (analog of the free product with amalgamation of groups). Let $A^{\prime}$ and $B^{\prime}$ be topological vector spaces containing a common dense set $S$. We define the union $A^{\prime} \cup B^{\prime}$ amalgamated over $S$ as

$$
A^{\prime} \cup{ }_{S} B^{\prime}=\left(A^{\prime} \oplus B^{\prime}\right) / C^{\prime} .
$$

Here $C^{\prime}=\{(c, c)\}$ is the completion of $S$ in the norms of both $A^{\prime}$ and $B^{\prime}$, that is, $c$ is a Cauchy filter of elements of $S$ in both $A^{\prime}$ and $B^{\prime}$ norms.

The reason for denoting the spaces by $A^{\prime}, B^{\prime}$ will become apparent later. We shall often drop the subscript $S$. 
If $A^{\prime}, B^{\prime}$ are the respective duals of $A, B$ then $A^{\prime} \cup_{S} B^{\prime}$ is the dual of the pairs $(a, b) \in A \oplus B$ satisfying $s \cdot a=s \cdot b$ for all $s \in S$.

We shall apply the above simple idea to density results. As we have seen in the above chapters we are unable to prove the general edge-of-the-wedge theorem when the dimension of the algebraic varieties exceeds 1 , so we shall be interested in density results for higher dimensional $V$. For simplicity we shall deal only with the cones $\Gamma^{ \pm}$ in $t$ space though there is no difficulty in extending the results to general cones.

Let us attempt to prove that the space of global solutions of $\mathbf{D} f=0$ is dense in the space of pairs $\left(f^{+}, f^{-}\right)$where $f^{ \pm}$is a solution in the tube over $\Gamma^{ \pm}$and the $f^{ \pm}$have equal Cauchy data.

We proceed along the lines of the proof of Theorems 1 and 2 of Part I. Consider the map $\alpha: f \rightarrow(f, f)$ of $\mathscr{E}$ into $\mathscr{E}^{+} \oplus \mathscr{E}^{\infty}$ where $\mathscr{E}^{ \pm}$is the space of $C^{\infty}$ functions on the tube over $\Gamma^{ \pm}$(closed cones). Suppose $\left(S^{+}, S^{-}\right)$is orthogonal to the image of $\alpha$ on solutions of $\mathbf{D}$. The adjoint $\alpha^{\prime}$ of $\alpha$ is defined by

$$
\alpha^{\prime}\left(S^{+}, S^{-}\right)=S^{+}+S^{-} \text {. }
$$

Thus by Fourier transform

$$
\hat{S}^{+}+\hat{S}^{-}=0 \text { on } V .
$$

Now, $\hat{S}^{ \pm}$belongs to $\hat{\mathscr{E}}^{ \pm \prime}(V)$ which is a space of entire functions on $V$ defined by growth conditions. Equation (6.3) means that

$$
\left.\hat{S}^{ \pm}\right|_{V} \in \hat{\mathscr{E}}^{+\prime}(V) \cup{ }^{\mathrm{a}} \hat{\mathscr{E}}^{-\prime}(V) .
$$

The symbol $A \cup{ }^{\text {a }} B$ refers to analytically uniform union meaning the space of entire functions satisfying the minimum growth conditions of $A$ and $B$ (see Part I for more details).

Suppose we knew that the growth conditions imposed by $\hat{\mathscr{E}}^{+\prime}(V) \cup^{\mathrm{a}} \hat{\mathscr{E}}^{-\prime}(V)$ are the same as those imposed by $\hat{\mathscr{E}}^{+\prime} \cup^{\mathrm{a}} \hat{\mathscr{E}}^{-\prime}$ on $V$. (The apparent paradoxical possibility that these spaces are distinct is clarified below.) Now using the fact that $\Gamma^{+} \cap \Gamma^{-}=\{0\}$, it is easily seen that $\hat{\mathscr{E}}^{+} \cup^{\mathrm{a}} \hat{\mathscr{E}}^{-\prime}$ is the tensor product of the space $\hat{\mathscr{E}}^{\prime}(Y)$ with polynomials in $\hat{t}$. This space is LAU in the sense of FA which implies that the functions satisfying the growth conditions of $\hat{\mathscr{E}}^{+\prime}(V) \cup^{\text {a } \hat{\mathscr{E}}^{-1}}(V)$ are the restrictions to $V$ of the functions in $\hat{\mathscr{E}}^{+\prime} \cup^{\text {a } \hat{\mathscr{E}}^{-\prime}}$.

All this means that

$$
\hat{S}^{ \pm}= \pm \hat{T} \text { on } V
$$

where $\hat{T} \in \hat{\mathscr{E}}^{+} \cup^{\mathrm{a}} \hat{\mathscr{E}}^{-\prime}$. Since $\hat{\mathscr{E}}^{ \pm \prime}$ is LAU

$$
\hat{S}^{ \pm}= \pm \hat{T}+\sum \hat{D}_{k} \hat{U}_{k}^{ \pm}
$$

where $\hat{U}_{k}^{ \pm} \in \hat{\mathscr{E}}^{ \pm \prime}$. By Fourier transform

$$
S^{ \pm}= \pm T+\sum D_{k} U_{k}^{ \pm} .
$$

We apply $\left(S^{+}, S^{-}\right)$to a pair of solutions $\left(f^{+}, f^{-}\right)$. We have

$$
\left(S^{+}, S^{-}\right) \cdot\left(f^{+}, f^{-}\right)=S^{+} \cdot f^{+}+S^{-} \cdot f^{-}=T \cdot\left(f^{+}-f^{-}\right) .
$$


Now, $T$ is an arbitrary distribution supported by $Y$ so the vanishing of $T \cdot\left(f^{+}-f^{-}\right)$ is equivalent to the vanishing of all derivatives of $f^{+}-f^{--}$on $Y$ which is equivalent to the vanishing of the Cauchy data of $f^{+}-f^{-}$on $Y$.

All this shows that we would know the density of global solutions if we knew that the growth conditions imposed by $\hat{\mathscr{E}}^{+\prime}(V) \cup^{\mathrm{a}} \hat{\mathscr{E}}^{-\prime}(V)$ were the same as those imposed by $\hat{\mathscr{E}}^{+\prime} \cup^{\mathrm{a}} \hat{\mathscr{E}}^{-\prime}$ on $V$.

In order to get our bearings on the meaning of this condition, consider the variety $V_{0}$ defined in $\left(\hat{t}_{1}, \hat{t}_{2}, \hat{y}\right)$ space by

$$
\hat{t}_{1}=i \hat{y}, \quad \hat{t}_{2}=-i \hat{y} .
$$

For any real $\hat{y}$ it is clear that $\operatorname{Im} \hat{t}$ does not lie in either $\hat{\Gamma}^{ \pm}$. The same is true for $\hat{y}$ near real. In fact we see readily that the topologies of both $\hat{\mathscr{E}}^{ \pm \prime}\left(V_{0}\right)$ are the same as that of $\hat{\mathscr{E}}^{\prime}\left(V_{0}\right)$ so $\hat{\mathscr{E}}^{+\prime}\left(V_{0}\right) \cup{ }^{\mathrm{a}} \hat{\mathscr{E}}^{-\prime}\left(V_{0}\right)=\hat{\mathscr{E}}^{\prime}\left(V_{0}\right)$ which is a far cry from $\left(\hat{\mathscr{E}}^{+\prime} \cup^{\mathrm{a}} \hat{\mathscr{E}}^{-\prime}\right)\left(V_{0}\right)$.

We see that the difficulty arises from points $(\hat{t}, \hat{y}) \in V$ for which $\operatorname{Im} \hat{t} \notin \hat{\Gamma}^{+} \cup \hat{\Gamma}^{-}$. Of course we could restrict our attention to varieties for which, at least on a sufficient set, $\operatorname{Im} \hat{t} \in \hat{\Gamma}^{+} \cup \hat{\Gamma}^{-}$. But such varieties seem to exist only in case of hypersurfaces (when the condition is clearly always satisfied) which were dealt with in Part I; or for some $V$ of dimension 1, which case was dealt with above; or for hyperbolic systems, in which case the edge-of-the-wedge theorem is easy.

Before showing how to solve this difficulty, we give another approach to density theorems, using the idea of union with amalgamation discussed at the beginning of this chapter. Instead of dealing with solutions $f$ directly, we deal with their Cauchy data, denoted by $C D(f)$. Since $f \rightarrow C D(f)$ is one-one, we give the $C D$ the topology to make this map a topological isomorphism. Call $A^{ \pm}$these $C D$ spaces and denote by $A^{ \pm \prime}$ their duals.

We want to determine if the $C D$ of global solutions are dense in those $C D$ which are $C D$ of both $f^{ \pm}$. Now the spaces $A^{ \pm \prime}$ both densely contain $\mathscr{E}^{\prime}(Y)^{r}$ where $r$ is the number of $C D$. Thus we can form the union with amalgamation

$$
A^{+,} \cup \mathscr{E}_{\mathscr{E}^{\prime}(Y)^{r}} A^{-\prime} \text {. }
$$

The dual of (6.10) is easily seen to be the space of common $C D$ of solutions $f^{+}$and $f^{-}$.

Suppose some $w=\left(w^{+}, w^{-}\right) \in(6.10)$ vanishes on the $C D$ of all global solutions. This is equivalent to saying it vanishes on the $C D$ of all exponential solutions. By tracing the definitions we find easily that this means

$$
\hat{w}^{+}+\hat{w}^{-}=0 \text { on } V .
$$

Here $\hat{w}^{ \pm}$is the Fourier transform of $w^{ \pm}$which is defined as follows: Each $w^{ \pm}$is defined by harmonic polynomials $h_{j}$ which define the Cauchy problem and elements $w_{j}^{ \pm}$which are distributions of a general sort on $Y$ in such a way that

$$
w^{ \pm} \cdot f^{ \pm}=\sum w_{j}^{ \pm} \cdot h_{j}(\partial / \partial t) f^{ \pm} \text {. }
$$

Then

$$
\hat{w}^{ \pm}=\sum \hat{w}_{j}{ }^{ \pm} h_{j}(\hat{t})
$$


That (6.11) implies that $\left(w^{+}, w^{-}\right)$is the zero element of $(6.10)$ is equivalent to the fact that $\pm \hat{w}^{ \pm}$is the limit in the topology of $\hat{\mathscr{E}}^{+\prime}(V) \cup{ }^{\mathrm{a}} \hat{\mathscr{E}}^{-\prime}(V)$ of elements in the

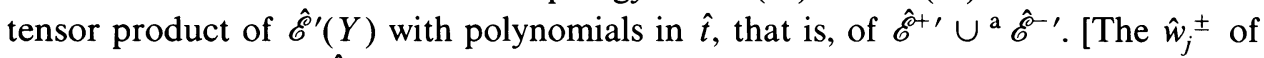
(6.13) may not be in $\hat{\mathscr{E}}^{\prime}(Y)$.]

At this point, one might think that we are at about the same point that we were in our first approach to density theorems. However, the present approach has one distinct advantage. For we can go beyond (6.11) by allowing different varieties $V^{ \pm}$ corresponding to different systems $\mathbf{D}^{ \pm} f^{ \pm}=0$.

What is the analog of (6.11)? As is seen in Chapter IX of FA, we can express the values $\hat{w}_{j}^{ \pm}(\hat{y})$ of $(6.13)$ in terms of the values of $\hat{w}^{ \pm}$at those points $\left(\hat{t}_{ \pm}^{k}, \hat{y}\right) \in V^{ \pm}$ lying above $\hat{y}$. These expressions are linear sums with coefficients which are algebraic functions of $\hat{y}$

$$
\hat{w}_{j}^{ \pm}(\hat{y})=\sum \hat{H}_{j k}^{ \pm}\left(\hat{t}_{ \pm}^{k}\right) \hat{w}^{ \pm}\left(\hat{t}_{ \pm}^{k}, \hat{y}\right) .
$$

Then (6.11) is replaced by

$$
\sum \hat{H}_{j k}^{+}\left(\hat{t}_{+}^{k}\right) \hat{w}^{+}\left(\hat{t}_{+}^{k}, \hat{y}\right)=\sum \hat{H}_{j k}^{-}\left(\hat{t}_{-}^{k}\right) \hat{w}^{-}\left(\hat{t}_{-}^{k}, \hat{y}\right) .
$$

Let us now explain how to derive density results.

We define the wedge set as the set of real $\hat{y}$ for which the set of $\hat{w}\left(\hat{t}_{+}^{k}, \hat{y}\right)$ for which $\operatorname{Im} \hat{t}_{ \pm}^{k}$ lie in $\hat{\Gamma}^{ \pm}$form a spanning set, meaning that the other values of $\hat{w}^{ \pm}\left(\hat{t}_{ \pm}^{k \prime}, \hat{y}\right)$ can be solved from (6.15) in terms of these $\hat{w}^{ \pm}\left(\hat{t}_{ \pm}^{k}, \hat{y}\right)$ for generic $\hat{y}$.

In particular, if $V^{+}=V^{-}$then the wedge set contains those $\hat{y}$ for which all $\hat{t}^{k}$ coordinates lie in either $\hat{\Gamma}^{+}$or $\hat{\Gamma}^{-}$.

THEOREM 6.1. Suppose that the convex hull of the wedge set (at infinity) is all real $\hat{y}$ space. Then entire $C D$ is dense in the space of those $C D$ which are $C D$ of solutions of $\mathbf{D}^{+} f^{+}$on the tube over $\Gamma^{+}$and of solutions of $\mathbf{D}^{-} f^{-}$on the tube over $\Gamma^{-}$.

Proof. It is a standard fact from the theory of functions of several complex variables that the indicator diagram of an entire function of exponential type is convex (see e.g. [11]). Now, our hypotheses combined with equations (6.15) imply that all the values $\hat{w}^{ \pm}\left(t_{ \pm}^{k}, \hat{y}\right)$ are of polynomial growth at infinity on the wedge set because the reciprocal of an algebraic function is generally large (see Chapter I of FA for details). Hence the same is true of the $\hat{w}_{j}^{ \pm}$. The above mentioned convexity then implies that the entire functions $\hat{w}_{j}{ }^{ \pm}$of exponential type are of zero exponential growth on the whole real $\hat{y}$ space. By examining the proof of the convexity we can easily strengthen it to prove that the $\hat{w}_{j}^{ \pm}$are actually of polynomial growth on the whole real $\hat{y}$ space. This shows that $\hat{w}_{j}^{ \pm} \in \hat{\mathscr{E}}^{\prime}(Y)$ which, by our above construction gives the density result which is Theorem 6.1 .

We can go further in the direction of Part I. Let $\xi$ be a continuous positive convex function on $\Gamma^{+}$which $\rightarrow \infty$ as $t \rightarrow 0$. The space $\mathscr{E}^{ \pm}(\xi)$ consists of all functions $f$ defined and $C^{\infty}$ in the interior of $\Gamma^{ \pm}$and such that for any $\varepsilon, A>0$, and any $l$

$$
\sup _{\substack{t \in \Gamma^{ \pm} \\|t|<A}}\left|f^{(l)}(t)\right| e^{-\xi(\varepsilon|t|)}<\infty .
$$

The topology of $\mathscr{E}^{ \pm}(\xi)$ is defined in the obvious manner. 
In order to prove the analog of Theorem 7 of Part I in the present situation we must assume that $\xi$ is a product of functions of one variable (see Examples 6 and 7 of Chapter V of FA). We assume, in fact that $\xi(t)=\tilde{\xi}\left(t_{1}\right) \cdots \tilde{\xi}\left(t_{m}\right)$. We can assert that $\hat{\mathscr{E}}^{ \pm}(\xi)$ is LAU. A function $F(\hat{t})$ belongs to $\hat{\mathscr{E}}^{ \pm \prime}(\xi)$ if and only if

$$
|F(\hat{t})| \leqslant c(1+|\hat{t}|)^{c} \begin{cases}\exp [c|\operatorname{Im} \hat{t}|] & \text { for } \operatorname{Im} \hat{t} \notin \hat{\Gamma}^{ \pm}, \\ \exp [-\eta(c \operatorname{Im} \hat{t})] & \text { for } \operatorname{Im} \hat{t} \in \hat{\Gamma}^{ \pm} .\end{cases}
$$

Here $\eta$ is the conjugate of $\xi$, that is,

$$
\eta(\hat{t})=\min _{t \in \Gamma^{ \pm}}[|\hat{t} \cdot t|-\xi(t)] .
$$

The AU structure is defined in the usual way.

We define the spaces $\mathscr{D}_{\xi}$ to consist of all $C^{\infty}$ functions $f(y)$ of compact support whose Fourier transforms satisfy

$$
|\hat{f}(\hat{y})| \leqslant c(1+|\hat{y}|)^{c} e^{c|\operatorname{Im} \hat{y}|-\eta(c|\operatorname{Re} \hat{y}|)} .
$$

The space $\mathscr{D}_{\xi}$ is given the natural AU topology. This makes $\mathscr{D}_{\xi}^{\prime}$ into an LAU space if it is nontrivial.

We can repeat the proof of Theorem 8 of Part I to deduce

Proposition 6.2. Suppose

$$
\int \frac{\eta(\hat{y})}{1+\hat{y}^{2}}<\infty
$$

and

$$
\eta(\hat{y}) \leqslant c|\hat{y}| .
$$

Then the kernel of $\mathbf{D}$ on $\mathscr{E}^{ \pm}(\xi) \otimes \mathscr{E}$ is contained in $\mathscr{E}^{ \pm} \otimes \mathscr{D}_{\xi}^{\prime}$.

We use the symbol $\otimes$ to denote the completed tensor product.

We point out only that inequality (35) of Part I should read $\eta(\hat{y}) \leqslant c|\hat{y}|$. The only changes in the proof are that the dichotomy $\operatorname{Im} \hat{t} \leqslant 0$ or $\operatorname{Im} \hat{t} \geqslant 0$ in equations (24) and (32) should be replaced by $\operatorname{Im} \hat{t} \notin \hat{\Gamma}^{+}$or $\operatorname{Im} \hat{t} \in \hat{\Gamma}^{+}$. Also the variable $x$ of Part I is denoted by $y$ here.

We can now repeat the proof of Theorem 6.1 to deduce (compare Theorems 8 and 10 of Part I).

THEOREM 6.3. Under the hypothesis of Proposition 6.2 the global solutions of D $f=0$ are dense in the pairs of solutions $f^{ \pm}$in $\mathscr{E}^{ \pm}(\xi) \otimes \mathscr{E}$ having equal Cauchy data. (The Cauchy data exists in $\mathscr{D}_{\xi}^{\prime}$.) A similar result holds for approximation to simultaneous $C D$ of $\mathbf{D}^{ \pm} f \pm=0$.

THEOREM 6.4. Suppose the integral in (6.20) diverges and that there exist sufficiently many vanishing curves $\beta$ so that a holomorphic function on $V$ which vanishes on all these $\beta$ vanishes on all of $V$. Then the Runge property holds, that is, any pair of solutions $f^{ \pm}$on $\mathscr{E}^{ \pm}(\xi) \otimes \mathscr{E}$ can be simultaneously approximated by global solutions. 
The definition of vanishing curve in the present situation is a refinement of that given in Part I. We define a vanishing curve $\beta$ as an irreducible algebraic curve lying in $V$ with the following properties

(i) The projection of $\beta$ on $\hat{t}$ is a Zariski open subset of a complex line $P$. Thus we may regard $\beta$ as a covering of $P$.

(ii) There is a piece $\tilde{\beta}$ of a branch of this covering whose projection $H$ is "essentially" a half-plane bounded by a line $L$ not parallel to the real space and such that for large $\hat{t} \in L$ we have $\operatorname{Im} \hat{t} \in \hat{\Gamma}^{+} \cup \hat{\Gamma}^{-}$.

(iii) $|\hat{y}| \leqslant c(1+|\hat{t}|)$ on $\tilde{\beta}$.

(iv) $|\operatorname{Im} \hat{y}| \leqslant c(1+|\hat{t}|)^{\gamma}$ for $\hat{t} \in L$ for some $\gamma<1$.

In case $V$ is elliptic the existence of vanishing curves depends on finding enough $\hat{y}$ which are essentially real for which $\hat{t} \in \hat{\Gamma}^{+} \cup \hat{\Gamma}^{-}$. Of course we can easily find such $\hat{y}$ for the Cauchy-Riemann variety but we have given examples above for which we cannot find such $\hat{y}$. For such systems we know very little about the Runge property.

REMARK. In case $\operatorname{dim} V=1$ we can extend the results of Chapter $V$ to the present situation.

Up to now we have been concerned with the AU union as this is related to density questions. Note, however, that when dealing with solutions of $\mathbf{D}^{ \pm} f^{ \pm}$on the tubes over $\Gamma^{ \pm}$for distinct $\mathbf{D}^{ \pm}$we introduced what may be regarded as a more subtle concept of AU union namely the dual of the space of common Cauchy data. More precisely, equations (6.11) or (6.3) imply that $\hat{w}^{ \pm}$or $\hat{S}^{ \pm}$belong to the $\mathrm{AU}$ intersection on $V$. Thus (6.15) can be regarded as a more subtle form of this. We think of (6.15) as an example of $\mathrm{AU}$ union defined via the relations $C D\left(f^{+}\right)=$ $C D\left(f^{-}\right)$.

We want to define the analog for $\mathrm{AU}$ intersection which is, in fact, more important to us. This is the $\mathrm{AU}$ intersection depending on relations (1.2).

We give the appropriate definitions; however our methods are not powerful enough to prove the desired results using the dual theory.

The principle underlying the definition of $\mathrm{AU}$ intersection in $\mathrm{FA}$ is that if $f$ belongs to several LAU spaces $W_{1}, \ldots, W_{l}$ then we want the weakest condition on the Fourier representation of $f$ to guarantee this. It is clear that the condition is

$$
f(x)=\int e^{i x \cdot \hat{x}} \frac{d \mu(\hat{x})}{k(\hat{x})}
$$

where $\mu$ is a bounded measure and for each $j=1,2, \ldots, l$ there is a $k_{j}$ in an $\mathrm{AU}$ structure for $W_{j}$ which is dominated by $k$.

This leads to the definition of the AU intersection

$$
W=W_{1} \cap{ }^{\mathrm{a}} W_{2} \cap{ }^{\mathrm{a}} \cdots \cap{ }^{\mathrm{a}} W_{l}
$$

as the $\mathrm{AU}$ space with $\mathrm{AU}$ structure $=\{k\}$ where

$$
k(\hat{x})=\max \left(k_{1}(\hat{x}), \ldots, k_{l}(\hat{x})\right)
$$

for any $k_{j} \in \mathrm{AU}$ structure for $W_{j}$. 
Putting things another way, $W$ is the largest AU space contained in all $W_{j}$.

If $V$ is an algebraic variety then we have defined $W(V)$ or $\hat{W}^{\prime}(V)$ using (6.24) for $\hat{x} \in V$. We sometimes write

$$
\hat{W}^{\prime}(V)=\hat{W}_{1}^{\prime}(V) \cap{ }^{\mathrm{a}} \cdots \cap^{\mathrm{a}} \hat{W}_{l}^{\prime}(V) .
$$

The AU union is defined in an analogous manner.

We want to find the weakest condition on the Fourier representation of each $f^{j}$ in consonance with relations of the form (1.2). If all $\mathbf{D}^{j}$ were the same that the blanket condition that $f^{j} \in W(V)$ where $W$ is the AU intersection defined above would be a sufficient condition for most reasonable relations. (Here we have fixed LAU spaces $W_{j}$ of functions or distributions on $\Omega^{j}$.) But this condition is generally too strong, especially when the number of relations in (1.2) is small.

We shall thus construct a new type of $\mathrm{AU}$ intersection, which depends on the relations (1.2). As such this intersection is not symmetric in all $j$ so we single out $j=1$ and search for the weakest growth condition on the Fourier representation of $f^{1}$ which guarantees the existence of $f^{2}, \ldots, f^{l}$ satisfying (1.2).

We could work in either the spaces $\hat{W}_{j}$ or $\hat{W}_{j}^{\prime}$. As mentioned in the introduction it seems that it is easier to give proofs in $\hat{W}_{j}$. However the definitions seem more natural in $\hat{W}_{j}^{\prime}$ so, in this chapter, we shall give the definitions in the framework of $\hat{W}^{\prime}$.

Let us think of $W_{j}$ as something like the space of $C^{\infty}$ functions on $\Omega^{j}$ which is assumed to be closed and contain $Y$ in its boundary. In this case each $W_{j}^{\prime}$ contains distributions of the form

$$
S_{0}=\sum h_{b}(\partial / \partial t) T_{b}
$$

where $T_{b}$ are distributions of compact support on $Y$. The uniqueness of the Cauchy problem tells us that such $\hat{S}_{0}$ are dense in each $\hat{W}^{\prime}(V)$.

In general we postulate the existence of a space $W_{0}$ of functions or distributions in $Y$ for which the set of sums of the form (6.26) with $T_{b} \in W_{0}^{\prime}$ can be thought of as the dual of the $\mathrm{AU}$ union $\mathrm{U}^{\mathrm{a}} W_{j}$. This dual is supposed injective and dense in each $W^{\prime}(V)$. This is our abstraction of the nature of the Cauchy problem and $Y$. It is via $W_{0}^{\prime}$ that relations (1.2) can be made workable.

To clarify our ideas, let us study relation (1.2) in case $l=2$. Applying $S_{0} \in W_{0}^{\prime}$ to (1.2) yields relations of the form

$$
{ }_{1} S_{0} \cdot f^{1}={ }_{2} S_{0} \cdot f^{2} .
$$

Here each ${ }_{j} S_{0}$ is of the form (6.26) so is in $\left(\mathrm{U}^{\mathrm{a}} W_{j}\right)^{\prime}$.

Relation (6.27) implies that if $S_{0}$ has the property that ${ }_{2} S_{0}$ is small in the topology of $W_{2}^{\prime}$ then ${ }_{1} S_{0} \cdot f^{1}$ is small. Putting this together with the fact that $f^{1}$ is continuous on $W_{1}^{\prime}$ means that $f^{1}$ is continuous on sums of the form

$$
S^{1}=S_{1}+{ }_{1} S_{0}
$$

where the sum is small if $S_{1}$ is small in $W_{1}^{\prime}$ and if there is a small ${ }_{2} S_{0}$ in $W_{2}^{\prime}$ satisfying (6.27). 
We can formulate things in slightly different terms: (6.27) is the precise way in which we use the relations (1.2) to transfer information from $W_{2}$ to $W_{1}$.

Situations like (6.28) were met in my treatment of gap theorems in Chapter XIII of FA. Since they involve topologies on $\Omega^{1} \cup \Omega^{2}$ they are manifestly not "convex" problems. Nevertheless we shall be able to treat them by Fourier analysis.

Since $\mathbf{D}^{j} f^{j}=0$ we can say more. Namely, $f^{j}$ is orthogonal to any $D_{k}^{j} S_{j}$ for $S_{j} \in W_{j}^{\prime}$.

Now, let us apply Fourier transform to (6.28) and the above. By the Fundamental Principle of FA we can factor by the module in $W_{1}^{\prime}$ generated by the $D_{k}^{1}$ by writing the Fourier transform of (6.28) as

$$
\hat{S}^{1}=\hat{S}_{1}+{ }_{1} \hat{S}_{0} \text { on } V_{1}
$$

where $V_{1}$ is the algebraic multiplicity variety defined by the $\hat{D}_{k}^{1}$.

As for the topology, we consider those ${ }_{1} \hat{S}_{0}$ for which the ${ }_{2} \hat{S}_{0}$ which corresponds to ${ }_{1} \hat{S}_{0}$ via (6.27) is small in the topology of $\hat{W}_{2}^{\prime}\left(V_{2}\right)$. Thus ${ }_{2} \hat{S}_{0}$ need not be small in all of $C^{n}$ or even on $V_{1}$; rather only on $V_{2}$. We are only interested in the corresponding values of ${ }_{1} \hat{S}_{0}$ on $V_{1}$ since that is all that is relevant. Thus the expression "small on $V_{1}$ " in (6.29) must be taken to mean that there is a splitting of $\hat{S}^{1}$ on $V_{1}$ into $\hat{S}_{1}$ and ${ }_{2} \hat{S}_{0}$ where each part has the appropriate smallness.

REMARK. We want to define an AU structure on $V_{1}$ in accordance with this idea. In defining functions $k$ we shall be guided by two principles.

(a) The functions $k$ that we need can be defined as the upper envelope of entire functions satisfying various conditions.

(b) When we express $k$ as a maximum of two different envelopes then we have in mind the heuristic principle (see B2 of the Introduction) the ability to split an entire function $\leqslant k$ into a sum of two appropriate entire functions.

Now (6.29) is a complicated condition since the norms governing $\hat{S}_{1}$ and ${ }_{1} \hat{S}_{0}$ are different. We might hope that (6.29) is equivalent to a growth condition on $V_{1}$. If that is so, then there is a natural growth condition which we could try. This is the AU intersection described in detail below but, roughly speaking, it is defined by $\max \left(\hat{S}_{1}\left(\hat{x}_{0}\right),{ }_{1} \hat{S}_{0}\left(\hat{x}_{0}\right)\right)$ the max being taken over all $\hat{S}_{1}$ and $\hat{S}_{0}$ for which $\left|\hat{S}_{1}(\hat{x})\right| \leqslant$ $k_{1}(\hat{x})$ on $V_{1}$ and $\left.\right|_{2} \hat{S}_{0}(\hat{x}) \mid \leqslant k_{2}(\hat{x})$ on $V_{2}$. Here $k_{1}$ and $k_{2}$ belong to AU structures for $\hat{W}_{1}^{\prime}$ and $\hat{W}_{2}^{\prime}$ respectively.

To delve more deeply into the problem, let us remark that there are, basically, two types of relations with which we can deal:

(i) Relations for which ${ }_{2} S_{0}=0$. (These occur in the above Example 3 where we require $f=0$ on $Y$.)

(ii) Relations for which ${ }_{2} S_{0} \neq 0$.

We thus modify (6.29) to

$$
\hat{S}^{1}=\hat{S}_{1}+{ }_{i} \hat{S}_{0}+{ }_{i i} \hat{S}_{0}
$$

where ${ }_{i} S_{0}$ correspond to case (i) and ${ }_{i i} S_{0}$ correspond to case (ii).

The problem we face is the following: Suppose that $\hat{S}^{1}$ is small in the topology of the AU intersection. We want to write $\hat{S}^{1}$ in the form (6.30) where $\hat{S}_{1}$ and ${ }_{i i} \hat{S}_{0}$ are small in their respective topologies but ${ }_{i} \hat{S}_{0}$ may be large. 
The terms ${ }_{i} \hat{S}_{0}$ and ${ }_{i i} \hat{S}_{0}$ are treated quite differently. To understand how things work it is better to write (6.30) in the form

$$
\hat{S}^{1}-{ }_{i} \hat{S}_{0}=\hat{S}_{1}+{ }_{i i} \hat{S}_{0} .
$$

The terms ${ }_{i} \hat{S}_{0}$ is used to make $\hat{S}^{1}-{ }_{i} \hat{S}_{0}$ small in certain parts of $V_{1}$. Then we want to split the left side into components $\hat{S}_{1}$ and ${ }_{i i} \hat{S}_{0}$.

Let us begin our precise treatment by writing (6.26) explicitly. We apply $\left\{S^{i}\right\} \in W_{0}^{\prime}$ to (1.2) and we obtain

$$
\sum_{j}\left(\sum_{b} e_{i j}^{b} \partial h_{b} S^{i}\right) \cdot f^{j}=0
$$

for all $i$. In accordance with our principle of distinguishing $W_{1}$, we rewrite (6.32) in the form

$$
\left(\sum_{b} e_{i 1}^{b} \partial h_{b} S^{i}\right) \cdot f^{1}=\sum_{j>1}\left(\sum_{b} e_{i j}^{b} \partial h_{b} S^{i}\right) \cdot f^{j} .
$$

Let $U^{i}$ be any distribution expressible in the form

$$
U^{1}=\sum_{i, b} a_{i} e_{i 1}^{b} \partial h_{b} S^{i}
$$

Then (6.33) gives us a certain amount of information regarding $U^{1}$. By Fourier transform we are led to study holomorphic functions $\hat{U}^{1}$ on $V_{1}$ which can be represented formally as

$$
\begin{aligned}
\hat{U}^{1}(\hat{t}, \hat{y}) \cdot \hat{f}^{1} & =\left[\sum_{i b} a_{i} e_{i 1}^{b} h_{b}(\hat{t}) \hat{S}^{i}(\hat{y})\right] \cdot \hat{f}^{1} \\
& =\sum_{j>1}\left[\sum_{i b} a_{i} e_{i j}^{b} h_{b}(\hat{t}) \hat{S}^{i}(\hat{y})\right] \cdot \hat{f}^{j} .
\end{aligned}
$$

We can interpret (6.35) as follows: Suppose we have a set of $\hat{S}^{i}(y)$ which can be divided in two parts. The set of $\hat{U}^{1}$ in the first part is bounded in the topology of $\hat{W}_{1}^{\prime}$ while for the second part, for every fixed $j>1$ the set of

$$
\hat{U}^{1 j}(\hat{t}, \hat{y})=\sum_{i b} a_{i} e_{i j}^{b} h_{b}(\hat{t}) \hat{S}^{i}(\hat{y})
$$

is bounded in the topology of $\hat{W}_{j}^{\prime}\left(V_{j}\right)$. Then $\hat{f}^{1}$ is bounded on the whole set of

$$
\hat{U}^{1}(\hat{t}, \hat{y})=\sum_{i b} a_{i} e_{i 1}^{b} h_{b}(\hat{t}) \hat{S}^{i}(\hat{y}) .
$$

It would be nice if there were enough relations (1.2). "Enough" means that every $U^{1} \in U^{a} W_{j}^{\prime}$ is of the form (6.34) or, what is essentially the same thing, that the functions $\hat{U}^{1}$ which appear on the left side of (6.35) form a dense set in $\hat{W}_{1}^{\prime}\left(V_{1}\right)$. This condition means that we have, essentially, enough conditions to determine all the Cauchy data of $f$.

Let us denote by $\mathbf{k}^{1}=\left(k_{2}, \ldots, k_{l}\right)$. Then we form the norm $\mathbf{k}_{V_{1}}^{1}$ on $V_{1}$ by setting (tentatively)

$$
{ }^{T} \mathbf{k}_{V_{1}}^{1}(\hat{t}, \hat{y})=\max \left|\hat{U}^{1}(\hat{t}, \hat{y})\right|
$$


the max being taken over all $\hat{U}^{1}$ of the form (6.37) for which (6.36) is bounded by $k_{j}(\hat{t}, \hat{y})$ for all $j>1$ whenever $(\hat{t}, \hat{y}) \in V_{j}$. We then define tentatively the AU norms $\tilde{k}^{T}$ on $V_{1}$ by

$$
\tilde{k}^{T}(\hat{t}, \hat{y})=\max \left(k_{1}(\hat{t}, \hat{y}),{ }^{T} \mathbf{k}_{V_{1}}^{1}(\hat{t}, \hat{y})\right) .
$$

The reason we use the word "tentatively" is that this definition is not suitable when the set of $U^{1}$ is not dense. The definition also suffers from being inexplicit in that it depends on the majorization $\left(6.38^{\mathrm{T}}\right)$ which seems difficult to compute explicitly.

We must, therefore, delve more deeply into the construction. We shall make a purely local ( $\hat{y}$ fixed) analog of $\left(6.38^{\mathrm{T}}\right)$. This local definition is completely explicit.

Let us denote by $d_{1}$ the dimension of the space of $\hat{U}^{1}(\hat{t}, \hat{y})$ of the form (6.37) for $\hat{y}$ fixed and generic. As $\hat{U}^{1}$ varies through this $d_{1}$ dimensional space the sums $\hat{U}^{1 j}$ of the form (6.36) vary through a linear space of, say, $d_{j}$ dimensions.

REMARK. We can interpret $d_{j}$ as the actual number of Cauchy data of $f^{1}$ that are determined by (1.2). This is the number of times we can use norms on the $U^{1 j}$ of (6.36).

We can now find a reasonable estimate for the $\hat{S}^{i}(\hat{y})$ or rather for $\sum_{i} a_{i} \hat{S}^{i}(\hat{y}) e_{i j}^{b}$ when $U^{1 j} \leqslant k_{j}$ on $V_{j}$. To see what this is, imagine the simplest case when $d_{j}(\hat{y})=1$ and all $e_{i j}^{b}=0$ except when $b$ corresponds to $h_{b} \equiv 1$. Then (6.36) is constant in $\hat{t}$ so $\sum a_{i} \hat{S}^{i}(\hat{y})$ being bounded by $k_{j}(\hat{t}, \hat{y})$ on $V_{j}$ above $\hat{y}$ means

$$
\left|\hat{U}^{1 j}(\hat{y})\right| \leqslant \min _{\hat{t}} k_{j}(\hat{t}, \hat{y}) \text {. }
$$

A heuristic argument when $d_{j} \geqslant 1$ leads to the estimate

$$
\begin{aligned}
\left|\sum a_{i} \hat{S}^{i}(\hat{y}) e_{i j}^{b}\right| & \leqslant\left(\text { polynomial) times }\left[d_{j} \text { th smallest } k_{j}(\hat{t}, \hat{y})\right]\right. \\
& =(\text { polynomial }) \text { times }{ }^{1} k_{j},
\end{aligned}
$$

that is, the min in (6.40) is replaced by the $d_{j}$ th term (meaning $\hat{y}$ and $j$ fixed, $\hat{t}$ varying) in increasing order of size, which is defined to be ${ }^{1} k_{j}(y)$. The polynomial factor plays no major role.

Given that (6.41) holds for every $j>1$, we want to know how large $\hat{U}^{1}$ can be on $V_{1}$. There are two cases

Case (i). $(\hat{t}, \hat{y})$ is not in any $V_{j}$ for $j>1$. Then, for algebraic reasons, the distance between $(\hat{t}, \hat{y})$ and $V_{j}$ should generally be greater than the reciprocal of a polynomial. This distance is of little importance and the value at $\hat{t}, \hat{y}$ is about the minimum of the ${ }^{1} k_{j}(\hat{y})$.

Case (ii). $(\hat{t}, \hat{y})$ belongs to some $V_{j}$ for $j>1$. For those $j$ it might happen that $\hat{U}^{1}$ is equal to some $\hat{U}^{1 j}$, for example if $e_{i j}^{b}=e_{i 1}^{b}$ for all $i, b$. Then the bound of $\hat{U}^{1}$ at that point would be $k_{j}(\hat{t}, \hat{y})$ instead of the minimum of the ${ }^{1} k_{j}(\hat{y})$.

Thus we are led to the correct definition of the norm.

$$
\mathbf{k}_{V_{1}}^{1}(\hat{t}, \hat{y})=\min _{j} \begin{cases}k_{j}(\hat{t}, \hat{y}) & \text { for }(\hat{t}, \hat{y}) \in V_{j} \text { and } \hat{U}^{1}=\hat{U}^{1 j} \\ { }^{1} k_{j}(\hat{y}) & \text { otherwise. }\end{cases}
$$


Before going further we want to comment on the usefulness of (6.38). We want to know that if $\left|\hat{U}^{1}\right| \leqslant \mathbf{k}_{V_{1}}^{1}$ on $V_{1}$ then $\left|\hat{U}^{1 j}\right| \leqslant k_{j}$ on every $V_{j}$. This is fine for the first option in (6.38), but how about the second option? From a somewhat sophisticated form of Proposition 2.1 we could deduce that the interpolation coefficients $\sum_{i} a_{i} e_{i 1}^{b} \hat{S}^{i}(\hat{y})$ are bounded by each ${ }^{1} k_{j}(\hat{y})$. If there are, in a suitable sense of linear dependence, more 1 relations than $j$ relations for $j>1$ then we could conclude, roughly, that $\left|\sum a_{i} e_{i j}^{b} \hat{S}^{i}(\hat{h})\right| \leqslant{ }^{1} k_{j}(\hat{y})$ for all $j$. Hence $\hat{U}^{1 j}$ is bounded by ${ }^{1} k_{j}$ on $V_{j}$ (except for unimportant factors). But $\hat{U}^{1 j}$ may not be bounded by $k_{j}(\hat{t}, \hat{y})$ on $V_{j}$ because if $d_{j}>1$ then for some $\hat{t}, \hat{y} \in V_{j}$ it may happen that $k_{j}(\hat{t}, \hat{y})$ is considerably smaller than ${ }^{1} k_{j}(\hat{y})$.

Thus we must make the

Assumption. For $\hat{t}, \hat{y}$ in the second option in (6.38)

$$
k_{V_{1}}^{1}(\hat{t}, \hat{y})=\min _{j,\left(\hat{t}^{\prime}, \hat{i}\right) \in V_{j}} k_{j}\left(\hat{t}^{\prime}, \hat{y}\right)
$$

The assumption means, essentially, that the right sides of (6.40) and (6.41) are the same.

Finally we make the

Definition. The AU intersection of the $W_{j}$ on $V_{1}$ using the relation (1.2) is given by the norms

$$
\tilde{k}^{\mathrm{a}}(\hat{t}, \hat{y})=\max \left(k_{1}(\hat{t}, \hat{y}), \mathbf{k}_{V_{1}}^{1}(\hat{t}, \hat{y})\right)
$$

with the proviso that for any $\hat{y}$ we cannot use $\mathbf{k}_{V_{1}}^{1}(\hat{t}, \hat{y})$ more than $d_{1}$ times in the $\max$. Thus if $\mathbf{k}_{V_{1}}^{1}(\hat{t}, \hat{y})$ exceeds $k_{1}(\hat{t}, \hat{y})$ at more than $d_{1}$ points, then we choose $d_{1}$ of them and use $\mathbf{k}_{V_{1}}^{1}$ at those points and $k_{1}(\hat{t}, \hat{y})$ at all other points above $\hat{y}$.

MAIN PROBLEM. Find conditions under which the relation (1.2) imply that $f^{1}$ belongs to this AU intersection.

The main point of these definitions is that the four examples given in the introduction can all be phrased as stating that functions satisfying equations on $\Omega^{j}$ and satisfying relations of the form (1.2) belong to the AU intersection of the spaces $\mathscr{E}\left(\Omega^{j}\right)$ depending on these relations. The equivalence of these two formulations can be proven without much difficulty.

Thus this admittedly complicated construction of AU intersection appears to be the correct formulation for the general problem of extensions of solutions of partial differential equations satisfying relations of the form (1.2).

\section{BIBLIOGRAPHY}

1. L. Ehrenpreis, Reflection, removable singularities, and approximation for partial differential equations, I, Ann. of Math. 112 (1980), 1-20.

2. __ Fourier analysis in several complex variables, Interscience, New York, 1970.

3. $507-509$.

4. __ Harmonic functions, in preparation.

5. The Radon transform and its ramifications, in preparation.

6. Analytically uniform spaces and some applications, Trans. Amer. Math. Soc. 101 (1961), $52-74$. 
7. __ Notes on group representations and differential equations, Berkeley, 1981.

8. D. Kinderlehrer, R. Spruck and L. Nirenberg, Regularity in elliptic free boundary value problems. I, J. Math. Anal. 34 (1978), 86-119.

9. H. Komatsu et al., Hyperfunctions and pseudo-differential equations, Lecture Notes in Math., vol. 287, Springer, 1973.

10. H. Lewy, On the reflection laws of second order differential equations in two independent variables, Bull. Amer. Math. Soc. 59 (1959), 37-57.

11. L. Ronkin, Introduction to the theory of entire functions of several variables, Transl. Math. Monographs, vol. 44, Amer. Math. Soc., Providence, R.I., 1974.

Department of Mathematics, Temple University, Philadelphia, Pennsylvania 19122 (Current address)

Department of Mathematics, Yale University, New Haven, Connecticut 06520 